\title{
Changing Relations Among Cognitive Abilities Across Development: Implications for Measurement and Research
}

\author{
Jala Rizeq
}

A Thesis submitted to the Faculty of Graduate Studies in Partial Fulfillment of the Requirements for the Degree of Master of Arts

\author{
Graduate Program In Psychology \\ York University, \\ Toronto, Ontario
}

June 2016

(C) Jala Rizeq, 2016 


\begin{abstract}
The constructs of intelligence and executive function are critical concepts of ability in neuropsychological research, cognitive research, developmental research, and clinical assessment. Yet, we have limited understanding of the changing age-related associations among these cognitive constructs. To better understand the development of these abilities, we compared a child sample and a young-adult sample on several measures of intelligence and executive functions. We used confirmatory factor analysis to estimate models for each developmental period. In addition, the association with ratings of inattention and hyperactivity/impulsivity, a dispositional measure of cognitive and behavioural regulation, was examined. The results indicated that cognitive abilities are more dependent on age in children than in young adults and that these abilities are more highly associated with ratings of cognitive and behavioural regulation in children than in young adults. The results support the integral relationship between intelligence and executive function throughout development, but especially in children.
\end{abstract}




\section{Acknowledgements}

Thank you to the many people who have contributed to my education and made this project possible. I would like to thank my supervisor Dr. Maggie Toplak for all her indispensible knowledge and consistent support at every stage. I would also like to thank my second committee member Dr. David Flora for his valuable comments and questions that pushed my thinking. I would like to acknowledge Dr. Doug McCann as my mentor throughout my years of study, and I am indebted for all his guidance and insight. Thanks as well to my fellow lab members for all their support. Thank you to my oral exam members, Dr. Christine Till and Dr. Dale Stevens for their intellectually stimulating feedback. Finally, thank you to my family and friends for their profound support, continuous encouragement, and investment in my education. 


\section{TABLE OF CONTENTS}

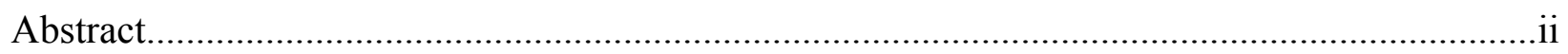

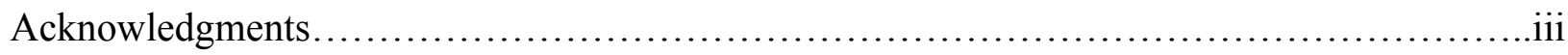

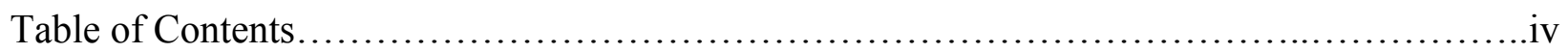

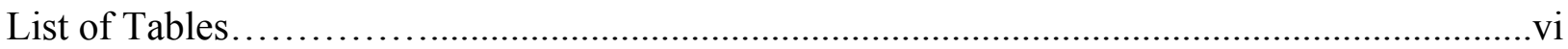

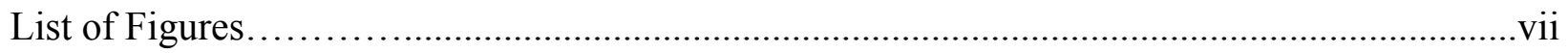

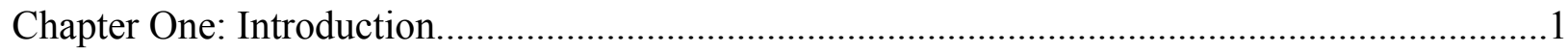

Executive Functions and Intelligence: Function and Development.....................................2

Association Among Intelligence and Executive Function Measures...................................7

Cognitive Abilities and Ratings of Inattention and Hyperactivity/Impulsivity.................10

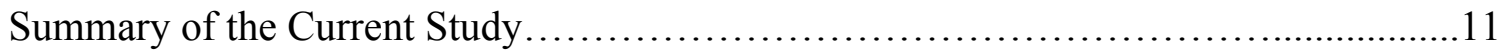

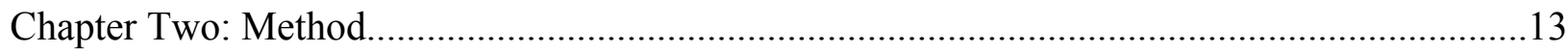

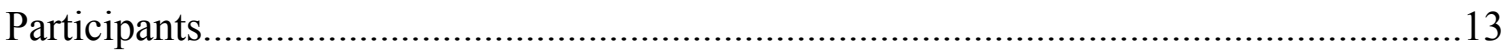

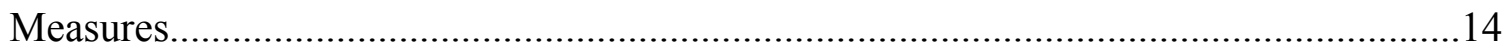

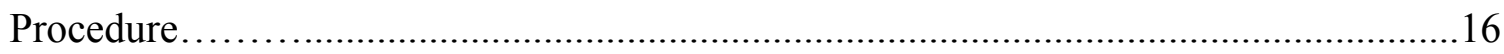

Data Analysis.....................................................................

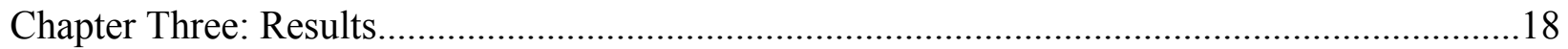

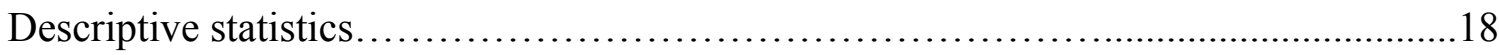

Age-Related Changes on Cognitive Measures......................................................19

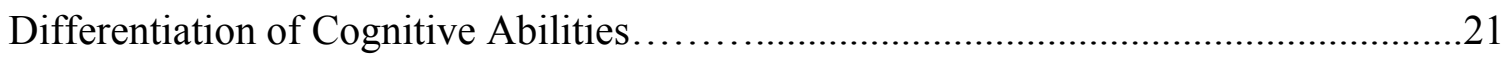

Predicting Ratings of Inattention and Hyperactivity/Impulsivity from Cognitive

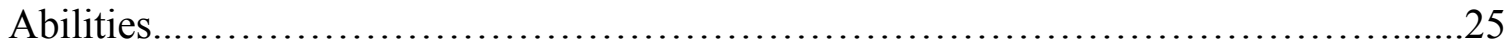

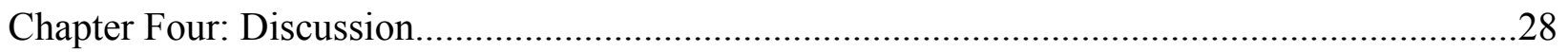

Associations of Intelligence and Executive Functions with Age........................................29

Differentiation of Cognitive Abilities........................................................................

Cognitive Processes Predictive of Ratings of Inattention and

Hyperactivity/Impulsivity ..................................................... 


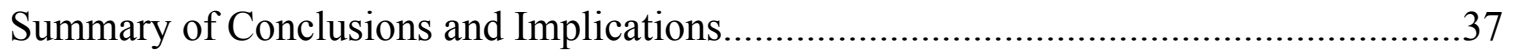

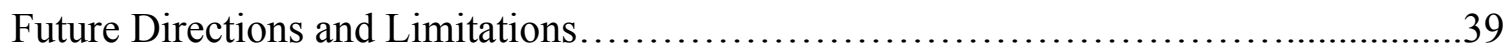

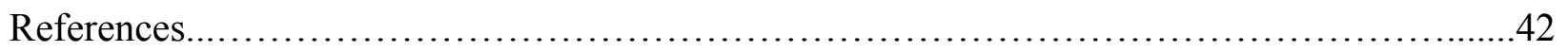




\section{LIST OF TABLES}

Table 1: Descriptive Statistics of Variables Used in the Child Sample.....................................19

Table 2: Descriptive Statistics of Variables Used in the Young-adult Sample.................19

Table 3: Correlations Among Intelligence, Executive Functions, and Parent

Inattention/Hyperactivity/Impulsivity Ratings in Child Sample...........................20

Table 4: Correlations Among Intelligence, Executive Functions, and

Inattention/Hyperactivity/Impulsivity Ratings in Young-adult Sample........................20

Table 5: Difference Between Correlation Coefficients Across the Two Samples...............21

Table 6: Fit of Cognitive Ability Indices CFA Models for Child and Young-adult Samples......23

Table 7: Percentage of Variance Explained in each Observed Variable by the Two Models

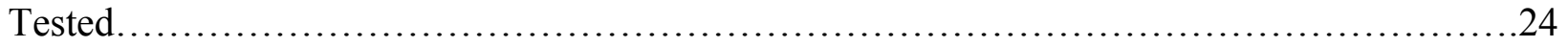

Table 8: Fit of Structural Models of Cognitive Abilities and Ratings of Inattention and

Hyperactivity/Impulsivity for Child and Young-adult Samples...............................26 


\section{LIST OF FIGURES}

Figure 1: Two Hypothesized Latent-factor Models of Cognitive Abilities...................... 10

Figure 2: Models A and B in the Child Sample........................................23

Figure 3: Models A and B in the Young-adult Sample.................................23

Figure 4: Structural Models A and B in the Child Sample..................................27

Figure 5: Structural Models A and B in the Young-adult Sample............................27 
Changing Relations Among Cognitive Abilities Across Development: Implications for

\section{Measurement and Research}

Individual differences in general intelligence are hypothesized to be driven by differences in executive function (EF) mechanisms (Benedek, Jauk, Sommer, Arendasy, \& Neubauer, 2014; Lezak, 2004), and some research has demonstrated strong associations between these constructs (Salthouse \& Davis, 2006). In younger populations, both intelligence and EF are heavily dependent on age explaining the strong relations between the two, yet some research still ignores the shared developmental component of these abilities or treats them as separate, unrelated cognitive constructs (see Dennis et al., 2009). With documented conceptual and experimental overlap between intelligence and EF processes, and age differences in younger populations, two important statistical and measurement-related considerations are noted: 1) whether to control for age effects in developing populations when examining the associations among cognitive abilities, and 2) whether to control for intelligence when examining the effects of EF on an outcome, or as the outcome, and vice versa (Arffa, 2007). Thus, the purpose of the current study was to examine the effects of age on performance-based cognitive measures of EF and intelligence in child and young-adult samples.

This study provided an important investigation of the associations among intelligence, $\mathrm{EF}$, and ratings of inattention and hyperactivity/impulsivity across age, within two different samples. Confirmatory factor analysis models were estimated in each of the two samples to better understand the underlying structure driving associations between measures of EF and intelligence. The relative contribution of EFs and intelligence in explaining ratings of inattention and hyperactivity/impulsivity in each developmental period was also examined as an illustration 
of the effects of statistical control when examining the effects of different cognitive abilities at two different periods of development.

\section{Executive Functions and Intelligence: Function and Development}

The interest in intelligence and EF grows from their role in a number of childhood disorders, including neurodevelopmental and cognitive-behavioural disorders like attentiondeficit/hyperactivity disorder (ADHD), autism, spina bifida meningomyelocele (SBM), and conduct disorder (Casey, Tottenham, \& Fosella, 2002; Dennis et al., 2009; Pennington \& Ozonoff, 1996). They are also associated with a number of developmental outcomes, as both intelligence and EF predict achievement (Biederman et al., 2004; Best, Miller, \& Naglieri, 2011; Borella, Carretti, \& Pelegrina, 2010; Protopapas, Archonti, \& Skaloumbakas, 2007; Mayes, Calhoun, Bixler, \& Zimmerman, 2009; Watkins, Lei, \& Canivez, 2007), an individual's performance on complex tasks (Miyake et al., 2000), and later health and success (Moffitt et al., 2011).

Executive Functions. EFs represent a number of top-down neurocognitive processes required for goal-directed behaviour (Benedek et al., 2014; Miller \& Cohen, 2001). These processes are important aspects of development that predict behaviours in everyday life (Best, Millet, \& Jones, 2009) and adaptive functioning in adolescence (Clark, Prior, \& Kinsella, 2002). EF is assessed using a number of performance-based measures. As these neurocognitive processes develop with age, children become increasingly competent in approaching problems, planning and organizing thoughts and behaviour, maintaining goals in mind and acting on them, and self-evaluation (Jurado \& Rosselli, 2007; Zelazo, Craik, \& Booth, 2004). The exact neurocognitive processes underlying EF are diverse and remain under investigation (Carlson, 
Zelazo, \& Faja, 2012). The dimensional structure of EF processes underlying performance on functional tasks, however, has received considerable attention in young adult and adult samples and more recently in children, with a focus on inhibition, updating, and set shifting (Miyake, Friedman, Emerson, Witzki, \& Howerter, 2000)

Inhibition or inhibitory control refers to the ability to control attention, thought, and behaviour in the presence of interfering internal or external stimuli, to overcome automatic impulses and respond appropriately so that with increasing inhibitory control, one is able to better restrict and regulate impulsive behaviours (Diamond, 2013; Miyake et al., 2000). Updating and monitoring of working memory representations, or simply updating, is a working memory operation that requires replacing old information with new information relevant to the task at hand (Jewsbury, Bowden, \& Strauss, 2015). The hallmark of updating is the active manipulation, rather than passive storage, of information (Miyake et al., 2000) in the multicomponent system of working memory that is needed to hold out-of-sight information in mind, manipulate it, and work with it to achieve goals and meet task demands (Baddeley \& Hitch, 1994; Baddeley, 2000; Engle, Tuholsko, Laughlin, \& Conway, 1999). The third core EF process which partially counts on both controlled attention and updating of working memory is set shifting, also known as cognitive flexibility, which describes one's ability to mentally shift from one task to another, utilizing alternative strategies, and processing more than one source of information (Zelazo et al., 2004). Set shifting is necessary for multitasking and for processing and managing several sources of information. It is usually measured by tests requiring switching between two timed tasks (Jewsbury et al., 2015).

Miyake et al.'s (2000) work with undergraduate student participants identified these three core separate, yet related, processes of EF: inhibition, set shifting, and updating. Support for the 
three-factor structure comes mostly from studies with youth and young adults (e.g.; Benedek et al., 2014; Friedman et al., 2008) and with children above the age of 10 (Brydges, Fox, Reid, \& Anderson, 2014) and 12 (Xu et al., 2013). It was also reported with children as young as 7 and 9 (van der Sluis, de Jong, \& van der Leij, 2007; Huizinga, Dolan, Maurits, \& van der Molen, 2006). Research with preschoolers, however, reports a one-factor model as the best fitting model (Weibe et al., 2011; Miller) or a two-factor model, depending on the tasks used (Geisbrecht, Müller, Mclnerney, \& Kerns, 2012). The structure of EF is influenced by the combination of observed cognitive variables used and the different periods of development it is estimated in. Taken together these results support the differentiation of these processes across age, starting with a general EF mechanism and developing with age into more specialized related entities.

Development of EF. Although the three main EF processes have slightly different developmental trajectories across childhood and adolescence, where inhibition seems to be the first to develop, followed by updating and lastly by set shifting, so far the research literature supports the age-related improvements in all EFs during those periods (Anderson, 2002; Zelazo, 2013; Zelazo et al., 2004; Zelazo \& Müller, 2002). Age-related changes are noticed in the increasing complexity of the rules and plans children and adolescents can keep in memory and utilize when needed for problem solving and their increasing ability to later reflect on them (Carlson et al., 2012). The sequencing of EF development is further dependent on the integrity, and is aligned with the maturation, of the prefrontal cortex (PFC) of the brain (Cunningham \& Zelazo, 2007; Miyake \& Friedman, 2012), which is a neuroanatomical correlate of major daily behavioural, emotional, and cognitive expectancies (Miller \& Cohen, 2001) and one that undergoes major development in adolescence (Gogtay et al., 2004). Thus, researchers agree that performance on EF measures depends on chronological age, especially in younger developing 
populations (i.e. early to middle childhood), and is mostly determined by a general ability that differentiates with age and experience (Carlson et al., 2012). With increasing age, however, performance on tasks of different EFs seems to decline at different rates (Jurado \& Rosselli, 2007; Salthouse \& Davis, 2006).

Intelligence. Intelligence, or intellectual functioning, like EF involves numerous mental abilities and is broadly defined through mental abilities which include, but are not limited to, reasoning, planning, problem-solving, and abstract thinking (Gottfredson, 1997). These same mental abilities are defined by Spearman's $g$ (general intelligence; Spearman, 1904) and are abilities required for successful performance on a variety of cognitive tasks (Duncan et al., 2000), which are usually assessed by psychometric tests like the Wechsler Abbreviated Scale of Intelligence (Wechsler, 1999). One of the seminal works on intelligence was Cattell's (1963; 1971) distinction between fluid and crystalized cognitive abilities that underlie performance on different types of cognitive tasks, where fluid ability usually underlies performance on nonverbal tasks and crystalized ability underlies performance on verbal tasks. According to Cattell, fluid general ability (Gf) represents complex mental abilities needed for reasoning and abstract thinking and reflects the capacity to apply one's skills and knowledge in novel situations and unfamiliar tasks. Next, crystalized general ability $(\mathrm{Gc})$ represents the set of skills and knowledge obtained through experience. According to McGrew (2009), "Gc is primarily a store of verbal or language-based declarative (knowing what) and procedural (knowing how) knowledge acquired through the investment of other abilities during formal and informal educational and general life experiences" (p. 5).

The Cattell-Horn extended Gf-Gc model of intelligence (Horn \& Noll, 1997) was integrated with Carroll's Three-Stratum model $(1993,1997)$ to form the Cattell-Horn-Carroll 
(CHC) model as a common structure for understanding and studying human cognitive abilities (McGrew, 1997, 2005; Sternberg \& Kaufman, 1998). Like other models and theories, the CHC remains under investigation (McGrew, 2009). However, an important aspect for this study was that the $\mathrm{CHC}$ model acknowledges the presence of a general cognitive ability $(g)$ as well as more specific cognitive abilities such as Gf and Gc.

Development of intelligence. In typically developing populations, Gf and Gc are highly associated in young children, but like the differentiation of EFs, the relations between fluid and crystallized intelligence indices are lower in adolescent, young adult, and middle adult groups (Li et al., 2004). Even when these two abilities differentiate, they are still related to a certain extent, depending on an individual's general intellectual ability. The rate of development of $g$ slows down around adolescence and the highest levels of performance on intelligence tests are reached by young adulthood (Anderson, 2001; Deary, Whalley, Lemon, Crawford, \& Starr, 2000). Despite the highly related tests of cognitive ability assessing intelligence and representative of general intelligence, the two aspects of intelligence examined in this study have slightly different age-related trajectories (Deary, Penke \& Johnson, 2010). Gf improves through childhood and peaks in adolescence while Gc continues to develop until early adulthood (Cattell, 1963). Whereas Gf shows declines with increasing age in later adulthood, Gc shows higher stability and less age-related decline (Cattell, 1963; Deary et al., 2010; Salthouse \& Davis, 2006).

It was expected that performance on the separate cognitive measures utilized in this study would improve with age in the child and adolescent sample due to the prime role of this developmental period in cognitive development, while no age-related effects were expected in 
the young-adult sample owing to the stability of mental abilities in early adulthood, consistent with previous findings (e.g., Deary et al., 2000; Salthouse \& Davis, 2006).

\section{Associations Among Intelligence and Executive Function Measures}

The positive relations among various measures of cognitive abilities have been noted as far back as Spearman's work (1904), with numerous examinations of common factors and general cognitive ability hypothesized to dictate performance on cognitive tasks (Tucker-Drob, 2009). Despite the strong connections between the conceptualizations of intelligence and EF constructs, the heterogeneity of the skills required for both and the diversity of the performancebased tasks have maintained the debate over the exact aspects of EF that are actually measured by intelligence tests (Ardila, Pineda, \& Rosselli, 2000; Friedman et al., 2006). The separation of the cognitive from the neuropsychology literature has also contributed to the separation of these constructs (Jewsbury, et al., 2015). Associations have been reported among various EF measures and intelligence indices (e.g., Arffa, 2007; Brydges et al., 2012). More specifically, updating has received the most attention among EF processes in relation with intelligence. The neuroscience literature supports the presence of shared prefrontal circuitry between tasks involving working memory (including the executive process of updating information), attention control during interference, and intelligence (Conway et al., 2003; Kane \& Engle, 2002; Nisbett et al., 2012), which further supports work that has found strong associations between updating and measures of Gf (Belacchi, Carreti, \& Cesare, 2010; Benedek et al., 2014; Engle et al., 1999; Salthouse \& Pink, 2008; Sbicigo, Piccola, Fonseca, \& Salles, 2013; Kane, Hambrick, \& Conway, 2005) and measures of general intelligence (Dang, Braeken, Ferrer, \& Liu, 2012; Friedman et al., 2006; Giofre, Mammarella, \& Carnoldi, 2013). Most of the working memory tasks require updating of new information for successful task completion, and thus relations between updating and Gf and 
general intelligence have been prominent in the literature. Other work in the neuropsychology literature has found associations between various performance-based EF measures and intelligence indices through factor analysis conducted to validate the EF construct and to estimate the dimensional latent abilities hypothesized to account for the relations among performance-based indices of cognitive and neuropsychological functioning (e.g., Brydges et al., 2012; Salthouse, Atkinson, \& Berish, 2003; Salthouse \& Davis, 2006).

Salthouse et al. (2003) found moderate to high associations between a latent EF variable and latent Gf, vocabulary, memory, and speed factors, the highest of which was with Gf. Salthouse and Davis (2006) report that their hypothesized latent EF variable predicted all EF indicators used (representative of inhibition, updating, and set shifting) and was related with latent cognitive ability variables of Gf and Gc. The relation between the EF construct and the Gf construct was the most notable across the three samples tested (child, student, and adult), in which the correlation between EF and Gf ranged from .87 to .98. Salthouse (2005) concludes that most of the measures utilized in assessing EF do not assess unique aspects above and beyond those accounted for by intelligence indices. In a sample of children between the ages of seven and nine, Brydges et al. (2012) found that a latent EF variable which loaded on nine measures of all three core EF processes accounted for $80 \%$ and $69 \%$ of the variance in Gf and Gc, respectively, concluding that EF is integrally related to both types of intelligence in children. This finding reflects the importance of examining relations among EF processes and general intelligence (Gf and Gc) in typically developing samples (Friedman et al., 2006).

Current directions. To date, concerns regarding research on the constructs of intelligence and EF and relationship between the two has been mostly carried out with young adult and adult samples. More work needs to be done to understand these relationships in 
children and adolescents (Brydges et al., 2012), especially because distinct cognitive abilities are not completely differentiated in these periods and depend heavily on age. One concern noted in the literature is contradictory findings regarding the association between EF and intelligence, with some studies reporting almost completely overlapping variance (e.g., Brydges et al., 2012; Salthouse \& Davis, 2006), while others reporting small or near-zero relations (e.g. Ardila et al., 2000; Rommelese et al., 2008). One difference in these studies is the scores used to indicate intelligence (i.e. IQ test scores), where Ardila et al. and Rommelese et al. use age-corrected intelligence scores with samples of children and adolescents, thereby removing much of the developmental variance determining EF and intelligence abilities in these periods of development. Thus, one needs to be aware of the discrepant approaches to measurement and analysis that can lead to divergent conclusions.

The literature presented so far clearly shows that the processes and skills assessed by EF measures include ones which are required for successful performance on intelligence indices (Anderson 2001; Diamond, 2013). With the well-documented associations and similar developmental trajectories of both intelligence and EF (e.g., Arffa, 2007; Conway, Kane \& Engle, 2003; Friedman et al., 2006; Salthouse \& Davis, 2006), this study examined the latent abilities or processes underlying performance on a sample of cognitive and EF measures in two distinct periods of development. The current work estimated a general cognitive ability model with one latent factor and a model with two latent factors to 1) test the differential effects of a general cognitive ability and two separate cognitive abilities on performance on EF and intelligence as currently measured, and to compare factor loadings across samples for a better understanding of the differentiation of these abilities across two different periods of development (childhood and young adulthood), and 2) illustrate how the use of age-corrected intelligence 
scores changes the results and conclusions in the child sample, but not in the young-adult sample. Two separate confirmatory factor analysis models were estimated to uncover the latent variables determining performance on five cognitive ability variables (i.e., measures of inhibition, updating, set shifting, nonverbal intelligence, and verbal intelligence) in two typically developing samples: a child sample and a young adult student sample. The two separate models that were tested are displayed in Figure 1.

Figure 1. Two hypothesized latent-factor models of cognitive abilities

A.

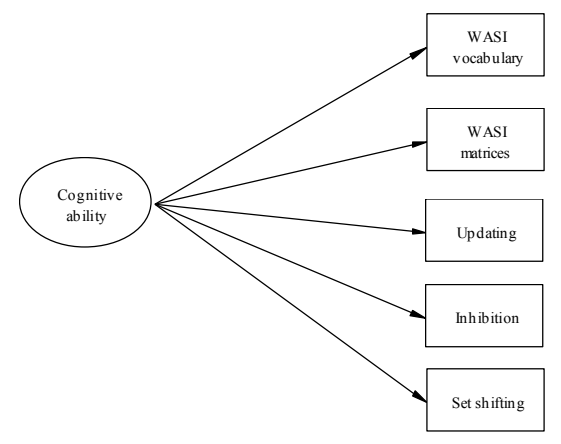

B.

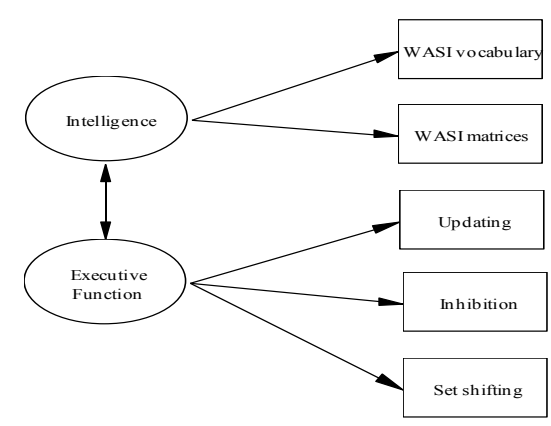

The literature presented supports an overlap between latent abilities required for performance on intelligence indices and EF measures which is reflected in the first model estimated, Model A, which is a simple one-factor model. Model B represents the distinction between intelligence and EF by including two separate, yet correlated latent variables as determinants of the cognitive abilities measured. These models have implications for understanding age-related associations between cognitive abilities, as well as understanding associations with other related constructs, such as cognitive and behavioural control.

\section{Cognitive Abilities and Ratings of Inattention and Hyperactivity/Impulsivity}

In order to further examine the association between intelligence and EFs, their unique as well as shared roles in explaining ratings of inattention and difficulties with 
hyperactivity/impulsivity were examined. Ratings of inattention and difficulties with hyperactivity/impulsivity have been associated with lower performance on EFs and intelligence measures in typically and atypically developing samples (Barkley, 1997; Diamond \& Lee, 2011; Gioia, Isquith, Guy, \& Kenworthy, 2000; Hofmann et al., 2012; Rajendran et al., 2013). More specifically, EFs play an important role in emotional and behavioural aspects of self-control (Gioia, Isquith, Guy, \& Kenworthy, 2000) and in the ability to provide content-appropriate responses (Pennington \& Ozonoff, 1996). Difficulties in controlling impulses and attention are considered one of the major concerns in clinical psychiatric populations (Strayhorn, 2002). Lower intelligence and EF scores and difficulties with self-control in children, such as higher impulsive behaviour, poor attention, and lack of persistency in carrying out tasks, are related to poor health outcomes, lower income, and higher rate of crimes later in life (Moffitt et al., 2011; Tangeny, Baumeister, \& Boone, 2004). In typically developing samples, achievement and performance at school, work, and everyday living situations has been related to EF and intelligence as well as self-control (Baumeister, 2002; Deary, Strand, Smith, \& Fernandes, 2007; Duckworth, Tsukayama, \& May, 2010; Duckworth, Quinn, \& Tsukayama, 2012; Jurado \& Rosselli, 2007).

Thus, the associations between EF and intelligence were used to predict ratings of inattention and hyperactivity/impulsivity, which were used as indicators of cognitive and behavioural control.

\section{Summary of the Current Study}

This investigation had three goals. First, correlations between age and performance on cognitive ability measures were examined, where higher positive associations were expected in 
the child sample than in the young-adult sample. Second, following previous research conducted to understand the underlying dimensions or processes of cognitive abilities (e.g., Brydges et al., 2012; Salthouse, 2005; Salthouse \& Davis, 2006), two confirmatory factor analysis (CFA) models of five distinct EF (updating, inhibition and set shifting) and intelligence (WASI matrices and WASI vocabulary) observed variables were estimated in each of the two developmental periods (see Figure 1):

A. a one-factor model of general cognitive ability;

B. a two-factor model of intelligence and EF, with three indicators for EF and two indicators of intelligence.

We expected that Model A would fit the data better in the child sample than in the young-adult sample and that Model B would have a better fit in the young-adult sample than in the child sample. It was also expected that higher amounts of variance would be explained in the observed cognitive measures in the child sample than in the young-adult sample. In estimating these models, age-corrected intelligence scores were also used as an alternative to uncorrected intelligence scores to statistically control for age in these models; using age-corrected rather than uncorrected intelligence scores was expected to alter the findings in the child sample but not in the young-adult sample.

The final goal was to expand models A and B to examine the extent to which the latent cognitive ability factors predict ratings of inattention and difficulties with hyperactivity/impulsivity. Specifically, the hypothesis was that neither intelligence nor EF from the two-factor model (Model B) in the child sample would predict these ratings because of the variance removed when assessing the effect of one factor while controlling the other, due to the particularly strong relations between EF and intelligence in that age group. On the other hand, it 
was expected that the general factor from Model A would predict self-control ratings in both the child and young-adult samples, because Model A preserves the shared processes between intelligence and $\mathrm{EF}$ needed for stronger prediction of inattention and hyperactivity/impulsivity ratings. The same models were retested using age-corrected intelligence scores to illustrate the heterogeneity of measurement and analysis utilized in the literature. Finally, the implications of the results on research and clinical work with developing populations are discussed.

\section{Method}

\section{Participants}

The current study used data from two samples. The first sample consisted of 250 children recruited from suburban and rural schools with 130 males and 120 females. Those children were recruited as part of a larger longitudinal research project and come from families of middle to upper socioeconomic status. Their ages ranged from 8 to 15 years $(M=10.66, S D=1.89)$. Four participants had pro-rated IQ scores lower than 80 and thus were excluded from the original sample of 254. There were 37 eight year-olds (19 males and 18 females), 50 nine year-olds (30 males and 20 females), 40 ten year-olds ( 22 males and 18 females), 23 eleven year-olds ( 15 males and 8 females), 51 twelve year-olds (16 males and 35 females), 33 thirteen year-olds (18 males and 15 females), 14 fourteen year-olds ( 9 males and 5 females), and 2 fifteen year-olds ( 1 male and 1 female).

The second sample consisted of 332 young adult students, with 92 males and 240 females. Their ages ranged from 17 to 25 years $(M=19.52, S D=1.92)$. To obtain a sample of young adults spanning the ages of 17 to 25 , fourteen participants who were above the age of 25 were excluded from the original sample of 346. Most of the young adult participants were 
recruited through an undergraduate student research portal and received course credit for participation, while others were paid for their participation. There were no gender or age differences between the two differentially recruited groups in the young-adult sample.

\section{Measures}

Ratings of inattention and hyperactivity/impulsivity. In the child sample, the Strengths and Weaknesses of ADHD-symptoms and Normal Behavior scale (SWAN; Swanson et al., 2005) was used to assess parent ratings of inattention and hyperactivity/impulsivity. The SWAN scale uses a strength-based formulation to assess inattention and hyperactive and impulsive behaviours on a continuum, with higher scores indicating better self-control (i.e., better controlled attention and behaviour). Parents were asked to rate their child's behaviour relative to same age peers for each of the 18 items using a seven-point scale ranging from far below average to far above average. Data were available from 211 parent reports. Coefficient alpha for this scale was 0.96 in the child sample.

In the young-adult sample, the Adult Self-Report Scale (ASRS) developed by the World Health Organization (WHO) was used to assess difficulties associated with AttentionDeficit/Hyperactivity Disorder (ADHD) in adults (Kessler et al., 2005). The scale has 18 items total with nine questions related to inattention, six questions related to hyperactivity, and three questions related to impulsivity. Participants reported the frequency with which they experienced these difficulties using a five-point Likert-type scale, with response categories of Never, Rarely, Sometimes, Often, and Very Often. Higher scores indicate more difficulties with inattention and hyperactivity/impulsivity. Coefficient alpha for this scale was 0.77 in the young-adult sample.

\section{Cognitive Ability Measures}


Verbal and nonverbal intelligence. The Vocabulary and Matrix Reasoning subtests from the Wechsler Abbreviated Scales of Intelligence (WASI; Wechsler, 1999) were used as verbal and nonverbal intelligence indices, respectively. These measures are reported to have high reliability and validity (Sattler, 2008). Higher raw scores on these indices indicate higher verbal and nonverbal intelligence. Higher age-corrected scores on these indices indicate better verbal and non-verbal intelligence compared to same-age peers.

Updating. In the child sample, the sentence span task was adapted from Gottardo, Stanovich, and Siegel (1996) to measure maintenance and updating operations of working memory. Children were asked to listen to sets of two to five statements and indicate whether each is true or false. The child was then asked to recall the last word of each sentence in the set. All statements contained familiar information to schoolchildren, were quite short (mean length 5.5 words, range 4 to 9 words), and grammatically simple (e.g, "cars have four wheels," "fish swim in the sky"). There were three 2-item sets, three 3-item sets, and three 4-item sets. Each child was asked to recall 27 words total. The updating score was the child's recall accuracy with higher scores indicating better updating.

In the young-adult sample, the Paced Auditory Serial Addition Test (PASAT; Gronwall, 1977) was used to assess updating. In this computer-administered task, single digits were serially presented at a rate of one digit every 3 seconds (Trial 1) and every 2 seconds (Trial 2). The participants were instructed to add each new digit to the one preceding it. Each trial was preceded by a practice trial. The total number of correct sums given in each trial was averaged to produce the updating score. Higher scores on this measure indicate better ability to update information in working memory. 
Inhibition. The Victoria version of the Stroop Test (Regard, 1981) was used to measure inhibition. In this task, participants had to name the incongruent font colour of colour words and resist the tendency to read colour words. Participants were presented with three different conditions, each containing 24 items. The first is a colour-naming condition, the second a wordnaming condition, and the third is an interference condition in which participants were asked to name the colour of the font in which the colour word was printed. The inhibition score of the Stroop task was calculated by subtracting the total naming time (in seconds) for the colour condition from the total naming time for the interference condition. More time required to complete the task indicates lower inhibition ability. Scores were reflected so that higher scores are indicative of better inhibition.

Set shifting. The Trail making Test Parts A and B (Reitan, 1958; 1995) were used to measure set shifting. Both parts of the test include 25 circles distributed on a sheet of paper. Part A asks participants to connect 25 numbered circles in ascending order. Part B asks participants to connect 12 lettered and 13 numbered circles, whereby the participant is instructed to alternate between numeric and alphabetic order, going from 1 to $\mathrm{A}$ to 2 to $\mathrm{B}$ to 3 to $\mathrm{C}$, and so on. Total completion time in seconds is recorded with higher time for completion indicating lower setshifting ability. To remove the effects of individual differences in processing speed, the set shifting score was obtained by removing the time taken to complete Part A from Part B. Scores were then reflected so that higher scores are indicative of better set shifting.

\section{Procedure}

Trained research assistants administered all testing with participants in both samples following similar orders of administration. Measures used in this study were part of a larger set 
of questionnaires and tests administered to both samples. In the child sample, parent consent and child assent were obtained before starting the study. The administration of task order in the child sample was as follows: demographics form, WASI Vocabulary, WASI Matrices, Stroop, Trail making, and Sentence Span. One parent completed the SWAN questionnaire for each child. In the young-adult sample, consent was obtained from all students before administration of the tasks. Task order was as follows: Demographics form, WASI Vocabulary, WASI Matrices, PASAT, Stroop, Trail making, and ASRS.

\section{Data Analysis}

The present analyses used three EF measures and two intelligence indices in each sample and a parent rating of inattention and hyperactivity/impulsivity scale in the child sample and a self-report rating scale of inattention and hyperactivity/impulsivity in the young-adult sample. All analyses were conducted separately for each of the two samples. There were no missing data in the young-adult sample, while there were 39 missing parents' ratings of inattention and hyperactivity/impulsivity in the child sample.

Before testing the hypotheses, the data was visually screened and followed up with descriptive statistics. To address the first goal, bivariate product-moment correlations were used to measure the linear associations among the measures of intelligence, EF, ratings of inattention and hyperactivity/impulsivity, and age in the two developmental periods. To address our second goal of cognitive ability differentiation, we tested the differences of the product-moment correlations between the two samples and used CFA to test the two potential factor structures hypothesized to underlie the five cognitive ability indicators: a) a one-factor model of cognitive ability (Model A); b) a two-factor model of intelligence and EF (Model B). Next, the third goal 
was addressed using structural equation modeling, expanding the same two CFA models by regressing ratings of inattention and hyperactivity/impulsivity on the different factors of each model.

All models were estimated using R software with the lavaan package (version 0.5-17; Rosseel, 2012). Maximum likelihood estimation was used with robust standard errors and fit statistics (Yuan \& Bentler, 2000) to adjust for multivariate non-normality. Full-information maximum likelihood was used to account for the missing SWAN ratings in the child sample. Model fit was evaluated using the standardized root mean square residual (SRMR), the root mean square error of approximation (RMSEA), the comparative-fit index (CFI), and the TuckerLewis index (TLI). Hu and Bentler (1999) recommend values lower than .08 for the SRMR and .06 for the RMSEA and a value close to .95 or above for CFI and TLI to indicate acceptable model fit. Less rigorous guidelines are also recommended where values of CFI and TLI greater than 0.90 (Kline, 2011) and values smaller than 0.08 for the RMSEA (MacCallum, Browne \& Sugawara, 1996) may represent acceptable fit.

\section{Results}

\section{Descriptive statistics}

The data were visually screened and the univariate distributions of all items were inspected as well as scatterplots of the bivariate distributions. Descriptive statistics are presented in Tables 1 and 2 for each of the child and young-adult samples' raw variable scores. The 39 children with missing parent reports of SWAN ratings in the child sample were compared to the rest of the sample on the five cognitive measures, with no notable differences. All variables' 
means for these 39 children were within the one standard error of the mean of the full sample of children with no missing data.

Table 1

Descriptive statistics of variables used in the child sample

\begin{tabular}{|c|c|c|c|c|c|c|c|}
\hline Variables & $n$ & Mean & Median & SD & $\begin{array}{c}\text { Range } \\
(\min , \mathbf{m a x})\end{array}$ & Skew & Kurtosis \\
\hline Age & 250 & 10.66 & 10.00 & 1.89 & 8,15 & 0.17 & -1.15 \\
\hline $\begin{array}{l}\text { Ratings of inattention and } \\
\text { hyperactivity/Impulsivity } \\
\text { Intelligence }\end{array}$ & 211 & 86.97 & 85.00 & 16.93 & 29,121 & -.09 & -0.32 \\
\hline WASI vocabulary & 250 & 42.47 & 43.00 & 7.51 & 25,62 & 0.11 & -0.45 \\
\hline $\begin{array}{l}\text { WASI matrices } \\
\mathbf{E F}\end{array}$ & 250 & 23.50 & 24.00 & 4.65 & 9,32 & -1.00 & 1.10 \\
\hline Updating & 250 & 22.08 & 21.00 & 5.86 & 8,40 & 0.80 & 0.08 \\
\hline Inhibition & 250 & 34.58 & 32.00 & 13.97 & 9,86 & 0.87 & 1.00 \\
\hline Set shifting & 250 & 63.70 & 55.00 & 41.33 & $-2,256.10$ & 1.66 & 4.01 \\
\hline
\end{tabular}

Table 2

Descriptive statistics of variables used in the young-adult sample

\begin{tabular}{lccccccc}
\hline Variables & $\boldsymbol{n}$ & Mean & Median & SD & $\begin{array}{c}\text { Range } \\
(\mathbf{m i n}, \mathbf{m a x})\end{array}$ & Skew & Kurtosis \\
\hline Age & 332 & 19.52 & 19.00 & 1.92 & 17,25 & 1.00 & 0.19 \\
$\begin{array}{l}\text { Ratings of inattention and } \\
\text { hyperactivity/Impulsivity }\end{array}$ & 332 & 51.89 & 51.00 & 8.60 & 29,76 & 0.25 & -0.07 \\
$\begin{array}{l}\text { Intelligence } \\
\text { WASI vocabulary }\end{array}$ & & & & & & \\
$\begin{array}{l}\text { WASI matrices } \\
\text { EF }\end{array}$ & 332 & 52.48 & 53.00 & 7.21 & 30,72 & -0.25 & -0.01 \\
Updating & 332 & 27.48 & 28.00 & 3.41 & 16,35 & -0.72 & 0.42 \\
$\begin{array}{l}\text { Inhibition } \\
\text { Set shifting }\end{array}$ & 332 & 38.49 & 38.75 & 9.10 & 10,57 & -0.35 & -0.27 \\
& 332 & 10.14 & 9.34 & 4.96 & $0.02,27.06$ & 0.78 & 0.54 \\
& 332 & 36.18 & 29.98 & 22.12 & -20.43, & 1.41 & 1.90 \\
\hline
\end{tabular}

\section{Age-related changes on cognitive measures}

The linear relationships among all variables were examined in each sample. Productmoment correlations among the variables are presented in Table 3 and 4 for the child and youngadult samples, respectively. 
Table 3

Correlations among intelligence, executive functions, and parent

inattention/hyperactivity/impulsivity ratings in child sample

\begin{tabular}{lccccccc}
\hline Measures & 1 & 2 & 3 & 4 & 5 & 6 & 7 \\
\hline 1. Age & 1 & .06 & $.57^{*}$ & $.35^{*}$ & $.24^{*}$ & $.48^{*}$ & $.36^{*}$ \\
$\begin{array}{l}\text { 2. Ratings of inattention and } \\
\quad \text { hyperactivity/impulsivity }\end{array}$ & & 1 & $.28^{*}$ & .13 & .09 & $.21^{*}$ & $.31^{*}$ \\
$\begin{array}{l}\text { Intelligence (raw scores) } \\
\text { 3. WASI vocabulary }\end{array}$ & & & & & & \\
4. WASI matrices & & 1 & $.47^{*}$ & $.40^{*}$ & $.45^{*}$ & $.40^{*}$ \\
EF & & & 1 & $.31^{*}$ & $.31^{*}$ & $.38^{*}$ \\
5. Updating & & & & & & $.32^{*}$ & $.37^{*}$ \\
6. Inhibition & & & & 1 & & $.39^{*}$ \\
7. Set shifting & & & & & & 1 \\
\hline
\end{tabular}

Note. ${ }^{*} p<.05$.

Table 4

Correlations among intelligence, executive functions, and inattention/hyperactivity/impulsivity ratings in young-adult sample

\begin{tabular}{lccccccc}
\hline Measures & 1 & 2 & 3 & 4 & 5 & 6 & 7 \\
\hline 1. Age & 1 & $-.13^{*}$ & .10 & .01 & .03 & -.02 & .06 \\
2. Ratings of inattention and & & 1 & -.08 & -.004 & -.06 & -.01 & -.02 \\
$\quad \begin{array}{l}\quad \text { hyperactivity/impulsivity } \\
\text { Intelligence (raw scores) }\end{array}$ & & & & & & \\
3. WASI vocabulary & & & 1 & $.17^{*}$ & $.18^{*}$ & $.16^{*}$ & $.11^{*}$ \\
4. WASI matrices & & & 1 & $.29^{*}$ & $.21^{*}$ & $.24^{*}$ \\
EF & & & & & & \\
5. Updating & & & & 1 & $.38^{*}$ & $.35^{*}$ \\
6. Inhibition & & & & & 1 & $.24^{*}$ \\
7. Set shifting & & & & & & &
\end{tabular}

In the child sample, all cognitive ability indices were significantly related with each other, with bivariate correlations ranging from $r=.31$ to $r=.47$. All five cognitive ability indices were also significantly related to age, ranging from moderate $(r=.24)$ to high $(r=.57)$ correlations. Performance on cognitive measures of verbal intelligence (WASI vocabulary), nonverbal intelligence (WASI matrices), updating, inhibition, and set shifting improved with age, meaning that older children had more developed cognitive abilities. Age, however, was not related to parent ratings of inattention and hyperactivity/impulsivity $(r=.06)$. Verbal 
intelligence, inhibition, and set shifting were significantly related to parent ratings of inattention and hyperactivity/impulsivity, with correlations ranging from $r=.21$ to $r=.31$.

In the young-adult sample, cognitive ability indices were significantly related to each other, ranging from small $(r=.11)$ to moderate $(r=.38)$ correlations. Age was only significantly related to ratings of inattention and hyperactivity/impulsivity with a small correlation of $r=-.13$. None of the cognitive ability indices was significantly correlated with ratings of inattention and hyperactivity/impulsivity or age.

\section{Differentiation of cognitive abilities}

First, the significance of the difference between bivariate correlation coefficients among intelligence and EF measures across the two samples was tested. Table 5 presents the z-value along with the significance value of each difference. Bonferroni correction was used to correct for the number of comparisons conducted; the adjusted alpha level was 0.005 .

Table 5

Difference between correlation coefficients across the two samples

\begin{tabular}{lcc}
\hline $\begin{array}{l}\text { Bivariate Association of Intelligence and EF } \\
\text { Indices compared }\end{array}$ & z-value & p \\
\hline WASI vocabulary - WASI matrices & 4.02 & .001 \\
WASI vocabulary - Updating & 2.87 & .0041 \\
WASI vocabulary - Inhibition & 3.84 & .0001 \\
WASI vocabulary - Set shifting & 3.72 & .0002 \\
WASI matrices - Updating & 0.26 & .7949 \\
WASI matrices - Inhibition & 1.28 & .2005 \\
WASI matrices - Set shifting & 1.84 & .0658 \\
Updating - Inhibition & -0.81 & .4179 \\
Updating - Set shifting & 0.27 & .7872 \\
Inhibition - Set shifting & 1.98 & .0477 \\
\hline
\end{tabular}

Note. Positive z-values indicate stronger relations in the child sample compared to the young adult sample

The relations among WASI vocabulary scores and all other cognitive and EF indices were significantly stronger in the child sample, all $p \mathrm{~s}<0.005$. Relations among WASI matrices 
scores and EF indices were not significantly different between samples, all $p \mathrm{~s}>0.005$. Among EF indicators, the relations were not significantly different across samples, $p s>0.005$. These results reflect the developing nature of cognitive abilities, such that as one gets older there is more room for differentiation between abilities underlying performance on measures of verbal intelligence and abilities underlying performance on measures of nonverbal intelligence and executive functioning. Nonetheless, the non-significant differences in relations of WASI matrices and other EF indices indicates that performance on these tasks is determined by similar skills, skills which are different from those needed for WASI vocabulary.

Underlying dimensions of cognitive abilities. Fit indices for the first set of CFA models estimated with each sample are reported in Table 6. In the child sample, both CFA models estimated had good fit statistics, but Model B (the two-factor model of EF and intelligence) had the best model fit statistics. Model B for the child sample is shown in Figure 2b. All completely standardized factor loadings reported in Figures $2 \mathrm{a}$ and $2 \mathrm{~b}$ are significant, $p<.001$. In the youngadult sample, both Model A (one-factor model of cognitive ability) and Model B fit the data well. All completely standardized factor loadings reported in Figures $3 \mathrm{a}$ and $3 \mathrm{~b}$ are significant, $p$ $<.001$. Note that the relationship between the latent factors in Model B was quite high in both samples (child sample $r=.90$; young-adult sample $r=.81$ ). The inter-factor correlation in the child sample was significantly stronger than in the young-adult sample with $\mathrm{z}=4.1, p<0.001$. Residual correlations were very small $(r<.05)$ for all models tested in both samples.

The scaled chi-square difference test of Bryant and Satorra (2012) was used to test whether there is a significant difference of model fit between the models in the two samples. The fit of Model A and the fit of Model B were not significantly different in the child sample, $\chi^{2}(1)$ $=2.33, p=0.13$. In the young adult sample, the fit of Model A was also not significantly 
different from the fit of Model B, $\chi^{2}(1)=1.21, p=0.27$. Thus, although Model B in the child sample displayed slightly better fit indices than Model A, the model fit difference was not statistically significant.

Table 6

Fit of cognitive ability indices CFA Models for child and young-adult samples

\begin{tabular}{lcccc}
\hline & \multicolumn{2}{c}{ Child } & \multicolumn{2}{c}{ Young-adult } \\
& Model A & Model B & Model A & Model B \\
\hline SRMR & 0.022 & 0.015 & 0.016 & 0.012 \\
RMSEA & 0.031 & 0.000 & 0.00 & 0.00 \\
CFI & 0.995 & 1.00 & 1.00 & 1.00 \\
TLI & 0.989 & 1.02 & 1.034 & 1.045 \\
AIC & 9158.0 & 9157.6 & 11290.6 & 11291.5 \\
BIC & 9210.8 & 9213.9 & 11347.7 & 11352.3 \\
\hline
\end{tabular}

Note. CFI Comparative Fit Index; TLI Tucker-Lewis Index; RMSEA Root Mean Square Error of Approximation; SRMR Standardized Root Mean Square Residual.

Figure 2. Models A and B in the child sample

a.

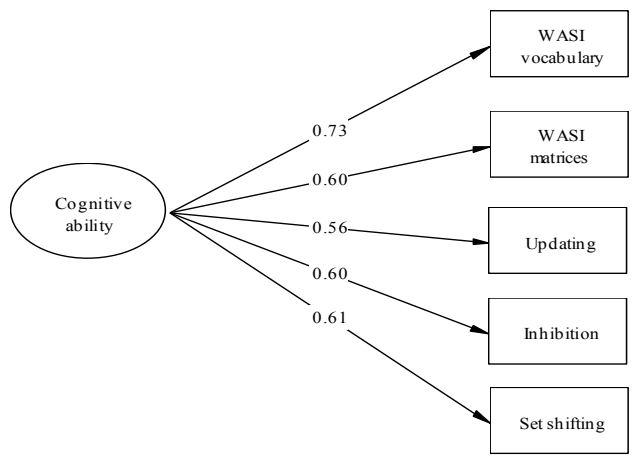

$\mathrm{b}$

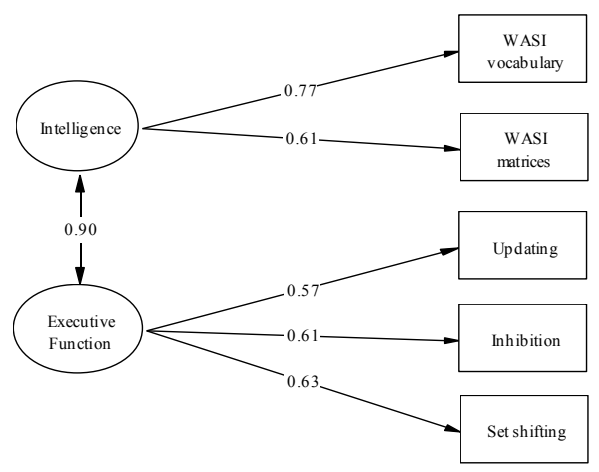

Figure 3. Models A and B in the young-adult sample

a.

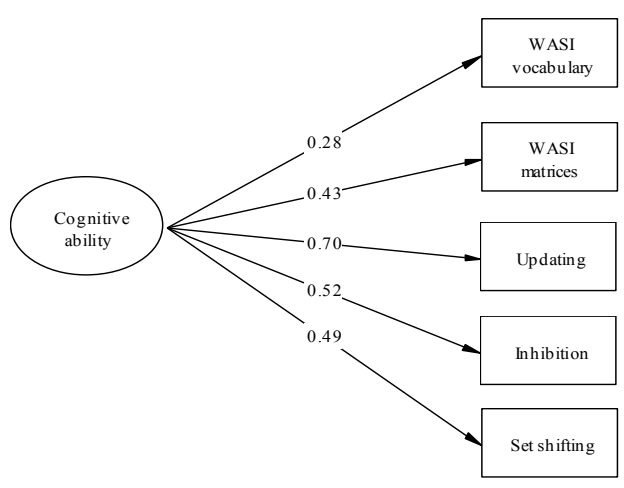

b.

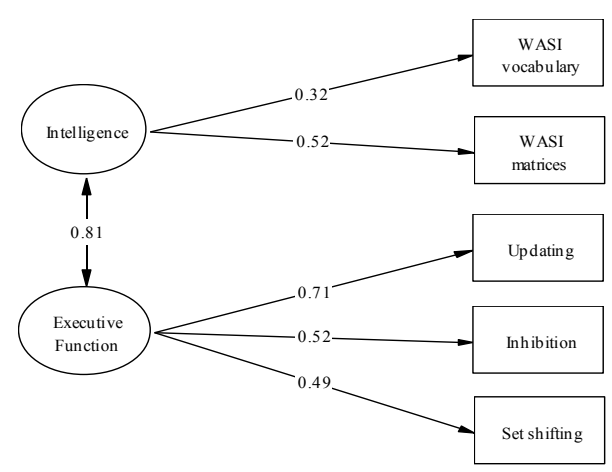


Table 7 reports the percentage of variance explained by the two models in each of the observed variables for each sample separately. These represent the amount of variance accounted for in each observed variable by the latent variables identified in the separate models tested. Both models in the child sample explain moderate to large amounts of variance in all observed intelligence and EF indices, ranging between $30.8 \%$ of the variance in updating explained by the general cognitive factor in Model A to $59.0 \%$ of the variance in WASI vocabulary explained by the intelligence factor in Model B. Models A and B in the young-adult sample also explain moderate amounts of variance in observed EF indices $(24.0 \%$ to $50.0 \%)$ and small to moderate amounts of variance in intelligence indices (7.8\% to $26.9 \%)$. Even though the results do not statistically differentiate among the models tested in the two samples, these results show the different patterns of relations across samples, with lower amounts of variance explained on performance measures of cognitive abilities in the young-adult sample than in the child sample. These findings indicate that performance on the different cognitive tasks is more highly determined by differentiated abilities than a general ability in the young-adult sample.

Table 7

Percentage of variance explained in each observed variable by the two models tested

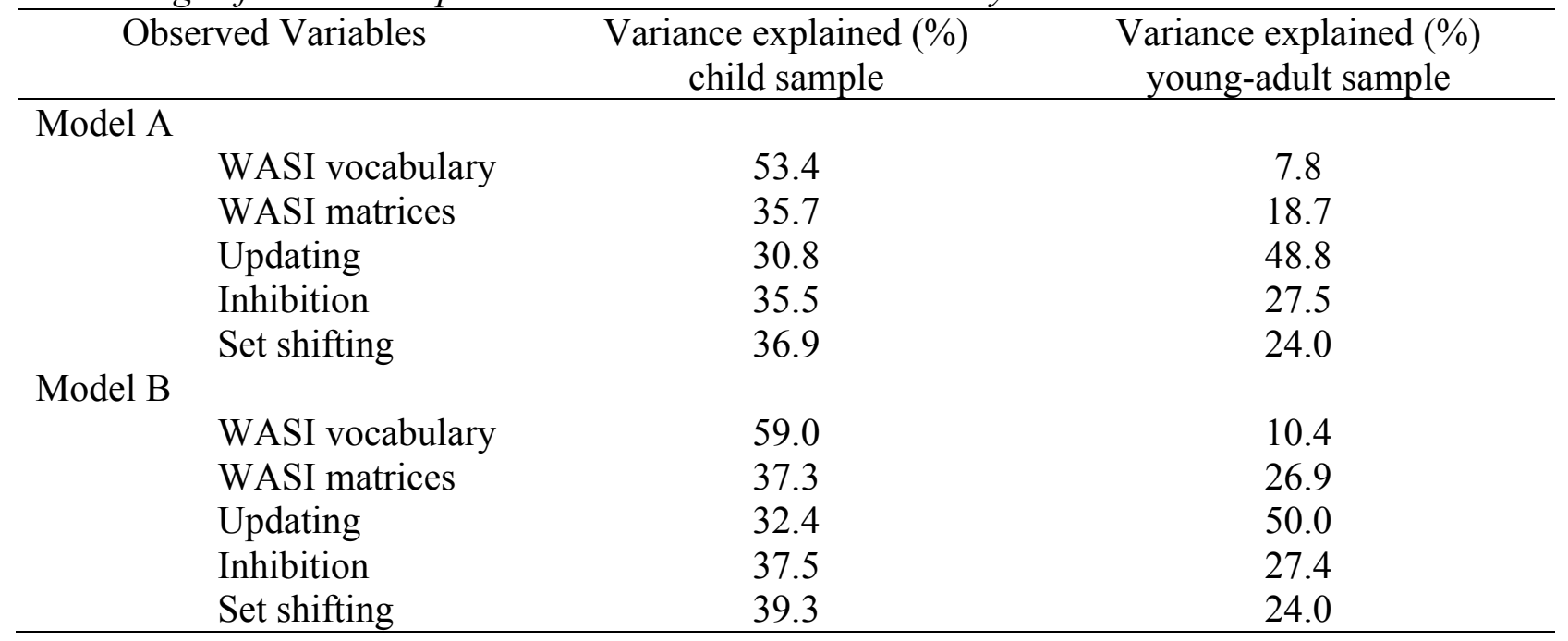


Controlling age effects in WASI scores. The same two models of the structure of cognitive abilities were re-estimated using the age-corrected WASI vocabulary and matrices scores. Because age-corrected intelligence scores remove the effects of age from the scores, as expected when utilizing the age-corrected WASI subtest scores in the child sample, the fit of Models A and B were considerably reduced (Model A SRMR $=0.10$, RMSEA $=0.22, \mathrm{CFI}=0.61$, and TLI $=0.21$; Model B SRMR $=0.04$, RMSEA $=0.09, \mathrm{CFI}=0.95$, and TLI $=0.87$ ). Model A fit the data poorly and thus was not interpreted, while Model B fit statistics were mediocre and relatively not as good as the fit statistics for Model B reported in Table 6. The two factors of intelligence and $\mathrm{EF}$ in Model B had a small positive association, $r=0.26, p=.004$. Thus, controlling age in the WASI subtest scores removed much of the shared variance between EF and intelligence measures, highlighting the developmental nature of these processes in this age group.

Also, as expected from the somewhat absent age effects in the young-adult sample (see Table 4), upon utilizing the age-corrected WASI subtests' scores the model fit statistics did not substantially differ from those presented in Table 6. Models A and B in the young-adult sample had good fit statistics (Model A SRMR $=0.02$, RMSEA $=0.00, C F I=1.00$, and TLI=1.02; Model B $\mathrm{SRMR}=0.01, \mathrm{RMSEA}=0.00, \mathrm{CFI}=1.00$, and $\mathrm{TLI}=1.04)$. The variance explained by each model in the observed cognitive ability indices was very similar to the values presented in Table 7. These results highlight the stability of these cognitive abilities and independence from any age effects.

\section{Predicting ratings of inattention and hyperactivity/impulsivity from cognitive abilities}

Next, structural models were estimated to examine the association between latent cognitive abilities and ratings of inattention and hyperactivity/impulsivity in both samples. 
Model fit indices for the set of SEMs estimated with ratings of inattention and

hyperactivity/impulsivity as an outcome are presented in Table 8 . Models A and B fit the data well, with similar fit indices, in both the child and young-adult samples.

Table 8

Fit of structural models of cognitive abilities and ratings of inattention and hyperactivity/impulsivity for child and young-adult samples

\begin{tabular}{lcccc} 
& \multicolumn{2}{c}{ Child } & \multicolumn{2}{c}{ Young-adult } \\
& Model A & Model B & Model A & Model B \\
\hline SRMR & 0.035 & 0.032 & 0.020 & 0.018 \\
RMSEA & 0.063 & 0.069 & 0.000 & 0.000 \\
CFI & 0.965 & 0.968 & 1.00 & 1.00 \\
TLI & 0.942 & 0.931 & 1.050 & 1.052 \\
AIC & 10935.9 & 10937.0 & 13665.6 & 13668.4 \\
BIC & 10999.3 & 11007.4 & 13734.1 & 13744.6 \\
\hline
\end{tabular}

CFI Comparative Fit Index; TLI Tucker-Lewis Index; RMSEA Root Mean Square Error of Approximation; SRMR Standardized Root Mean Square Residual

In the child sample, the two latent variables of intelligence and EF identified in Model B did not significantly predict parents' ratings of inattention and hyperactivity/impulsivity (intelligence $\mathrm{B}^{*}=0.01, p=0.98 ; \mathrm{EF} \mathrm{B}^{*}=0.34, p=0.37$; see Figure $4 \mathrm{~b}$ ). The cognitive ability latent variable from Model A, however, did significantly predict parents' ratings of inattention and hyperactivity/impulsivity, with $\mathrm{B}^{*}=0.34, p<0.001$ (see Figure 4a). Model A explained $11.6 \%$ of the variance in parents' ratings of inattention and hyperactivity/impulsivity, while Model B explained $12.2 \%$ of the variance in parents' ratings of inattention and hyperactivity/impulsivity.

In the young-adult sample, latent variables of intelligence and EF from Model B did not significantly predict ratings of inattention and hyperactivity/impulsivity, $p=0.83$ and $p=0.97$, respectively (see Figure 5b). The cognitive ability latent variable in Model A also did not significantly predict ratings of inattention and hyperactivity/impulsivity, $p=0.37$ (see Figure 5a). 
Model A explained $0.4 \%$ of the variance in ratings of inattention and hyperactivity/impulsivity, and Model B explained 0.5\%.

Figure 4. Structural Models A and B in the child sample

a.

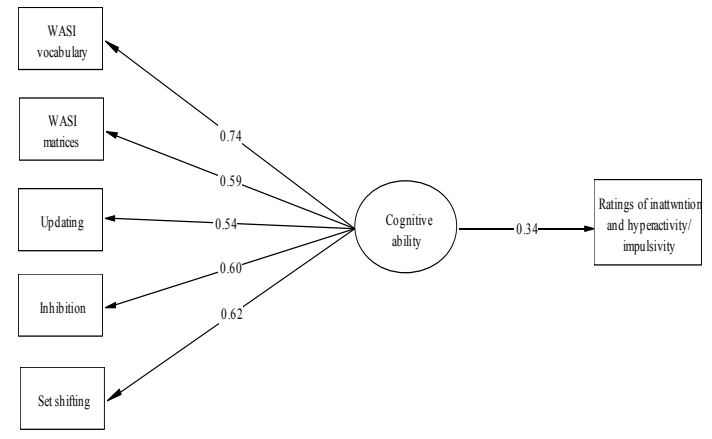

b.

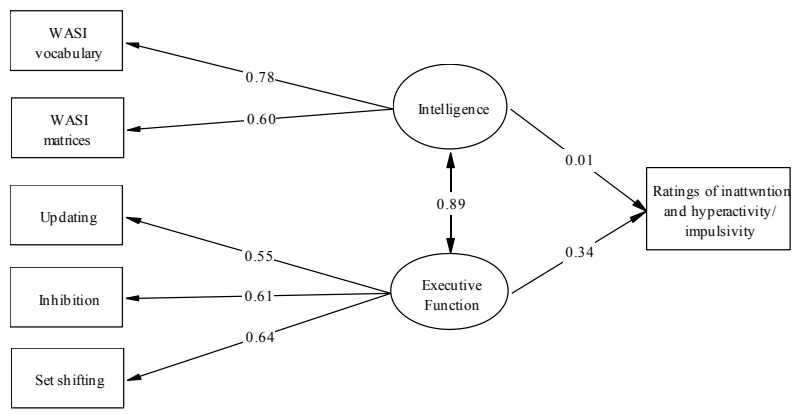

Figure 5. Structural Models A and B in the young-adult sample

a.

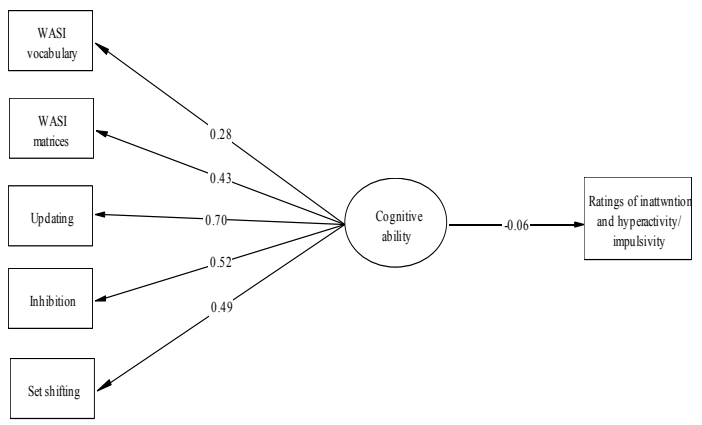

b.

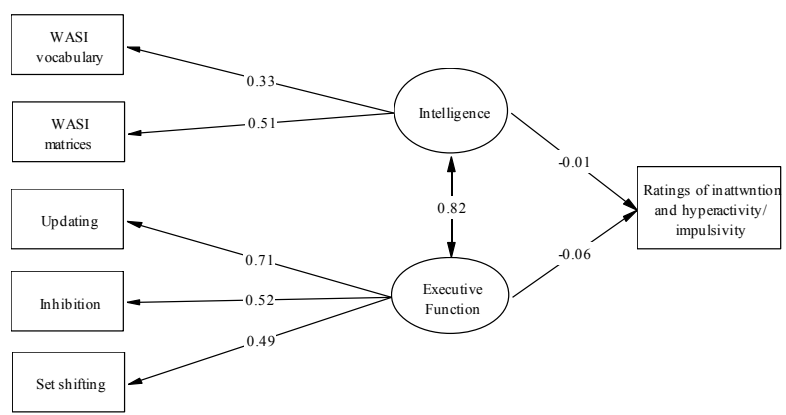

Controlling age effects in WASI scores. The same models in both samples were again reestimated using age-corrected WASI vocabulary and matrices scores. As expected, in the child sample the models' fit statistics changed. Model A fit statistics were considerably reduced to indicate poor fit $(\mathrm{SRMR}=0.09, \mathrm{RMSEA}=0.18, \mathrm{CFI}=0.59, \mathrm{TLI}=0.32)$ and thus its parameter estimates were not interpreted. Model B fit statistics were worse than those reported earlier and were within the mediocre fit range $(\mathrm{SRMR}=0.05, \mathrm{RMSEA}=0.10, \mathrm{CFI}=0.91, \mathrm{TLI}=0.80)$. The correlation between the EF and intelligence factors was reduced to $r=.20$ and both factors significantly predicted parent ratings of inattention and hyperactivity/impulsivity (Intelligence 
$\left.\mathrm{B}^{*}=0.24, p<0.001 ; \mathrm{EF} \mathrm{B}^{*}=0.31, p=0.002\right)$. These results indicate that when the variance due to age was removed from the intelligence indices, the relation between EF and intelligence changed substantially. The one-factor model was no longer adequate and the extent of reduction in the correlation between intelligence and EF factors in the two-factor model lead to both factors significantly predicting ratings of inattention and hyperactivity/impulsivity, which was not the case in the previous analysis where raw WASI scores were used.

In the young-adult sample, fit statistics for Models A and B did not change from results reported in Table 8, and both models fit the data well (Model A SRMR=0.02, RMSEA=0.00, $\mathrm{CFI}=1.00, \mathrm{TLI}=1.05 ;$ Model B SRMR=0.02, RMSEA=0.00, CFI=1.00, TLI=1.06). The correlation between intelligence and EF factors in Model B remained high, with $r=.78$, which speaks to the stability of cognitive abilities in this developmental period where very little, if any, age effects are present and thus neither the model fit statistics nor the correlation between the cognitive ability factors was influenced. None of the factors in the two models significantly predicted ratings of inattention and hyperactivity/impulsivity, which was also expected.

\section{Discussion}

The present study examined the developmental associations among different indices of cognitive abilities (intelligence and EF) in a child and a young-adult sample. The results were consistent with the predictions of this study and with previous work and offer methodological contributions for the assessment of cognitive abilities in childhood and young adulthood. The results included 1) an examination of the associations among five intelligence and EF indices with age, 2) comparison of relations among cognitive indices between samples, 3) models of latent cognitive abilities for each sample, and 4) structural models of latent cognitive abilities 
predicting ratings of inattention and hyperactivity/impulsivity as an illustration of the implications of statistical control for examining developing cognitive abilities. The results supported the age-related effects in the child sample and the absence of these effects in the young-adult sample, as well as a pattern of stronger relations among cognitive abilities in the child sample compared to the young-adult sample. Further, the latent cognitive abilities identified explained higher amounts of variance in the child sample compared to the young-adult sample, and the relations between latent abilities with ratings of inattention and hyperactivity/impulsivity also differed depending on the sample.

\section{Associations of Intelligence and Executive Functions with Age}

Age was significantly positively related to all cognitive measures in the child sample including WASI vocabulary (indicator of verbal intelligence), WASI matrices (indicator of nonverbal intelligence), and indices of updating, inhibition, and set shifting. More specifically, the association between WASI vocabulary and age was stronger than that between WASI matrices and age. This finding is in line with our understanding of verbal intelligence that depends on Gc and corresponds to verbal skills, instruction, and knowledge acquired through education, and thus is more dependent on knowledge accumulated throughout the years of development (Skirbekk, 2004). Regarding EF indices, inhibition had the strongest relationship with age followed by set shifting and updating, all of which were positive and significant, also in line with the reported age improvements in childhood and adolescence (Carlson et al., 2012; Lehto, Juujärvi, Kooistra, \& Pulkkinen, 2003). Cognitive ability indices were all significantly, positively correlated with each other, with moderate to high correlations. It is important to note that these reported relations were between the raw scores, rather than age-corrected scores, of the WASI vocabulary and WASI matrices subtests and EF measures. 
Results from the young-adult sample were quite different than those from the child sample. Age was not significantly related to any of the intelligence or EF indices. This finding reflects the stability of intellectual abilities and EF in adulthood, consistent with previous research with young-adult samples (Deary et al., 2000; Salthouse \& Davis, 2006). These results are also consistent with the rate of maturation of the PFC that continues developing until late adolescence (Gogtay et al., 2004), which has been related to the development of cognitive abilities and performance on both intelligence and EF measures (Cunningham \& Zelazo, 2007; Nisbett et al., 2012). It seems then that the development of cognitive abilities in childhood and adolescence, as opposed to young adulthood, is heavily influenced by chronological age. The relations among cognitive abilities also differ between childhood and young adulthood, as discussed below.

\section{Differentiation of Cognitive Abilities}

While there is debate over the multifarious construct of EF, there is literature supporting the three-factor EF model that stemmed from Miyake et al. (2000). When EFs are measured alongside other cognitive indices, such as intelligence, however, there is substantial overlap between these constructs (e.g., Arffa, 2007; Brydges et al., 2012; Jewsbury, et al., 2015; Salthouse et al., 2003). Considering these strong associations, and the importance of these constructs in formal assessments, this study examined the patterns of relations among cognitive abilities across samples, elucidating the differentiation of these abilities between different age groups (i.e. across the two samples) and by comparing the amount of variance explained in performance on EF and intelligence indices by latent cognitive abilities. 
All of the associations among the cognitive indices in the child sample were stronger than those in the young-adult sample. Specifically, the scores of the child sample on measures of a variety of cognitive abilities were highly related and more strongly related with each other than within the young-adult sample. However, the relations between WASI matrices and all other EF indices were not significantly different between samples. This result is consistent with previous work by Salthouse and colleagues $(2003 ; 2006)$ where very high correlations were reported between measures of nonverbal intelligence with EF measures across all age groups. Further, relations among EF indices did not significantly differ across samples, which indicates that these relations and ones between EF indices and WASI matrices are not necessarily a function of age differences but rather might be based on shared underlying cognitive abilities. The next set of analyses estimated models with latent cognitive abilities to further represent their contribution to performance on cognitive indices at different ages.

Child sample. Both the one-factor model (Model A) and the two-factor model (Model B) with three indicators loading on an EF factor (updating, inhibition, and set shifting) and two indicators loading on an intelligence factor (WASI vocabulary and WASI matrices) yielded good fit statistics, and the fit of these models was not statistically different. The inter-factor correlation in Model B was very high $(r=.90)$ indicating that these abilities are highly overlapping and a more parsimonious one-factor model of a general cognitive ability is a more appropriate representation of the relations among the cognitive indices in this sample. This high inter-factor correlation is not surprising considering reported findings (Salthouse \& Davis, 2006) and are even stronger than ones found in Brydges et al. (2012), raising concerns regarding the EF construct (Salthouse, 2005) or its measurement (Pennington \& Ozonnoff, 1996). However, these results are also expected due to the dependence on age in the development of these abilities 
among children. Models A and B explained moderate to large amounts of variance in each of the five observed variables. The finding that even the one-factor model of general cognitive ability explained similar amounts of variance in the observed variables to that explained by the two factors of EF and intelligence further supports the notion of the presence of a general cognitive ability that is needed to perform well on distinct cognitive measures in this sample, including both EF and intelligence measures.

Then, models were re-estimated using age-corrected WASI scores. As expected, the models with age-corrected WASI subtest scores yielded results that differed from age-corrected scores, with considerable changes in model fit statistics. The most notable difference was that the one-factor model no longer had a good fit to the data and the two-factor model had mediocre fit statistics with a small inter-factor correlation between EF and intelligence, in contrast to the large correlation between the factors in the model with raw WASI subtest scores. This finding emphasizes the role of age in childhood and adolescence in determining the relation between EF and intelligence.

Young-adult sample. The two models estimated in the young-adult sample fit the data well and, similar to the child sample, model fit did not significantly differ across the two models. However, the inter-factor correlation between EF and intelligence in Model B $(r=.81)$ was smaller than that in the child sample, yet the correlation between EF and intelligence was still large. These results suggest that there might be a domain-free general cognitive ability underlying performance on intelligence and EF indices among young adults, causing performance on different measures to correlate with each other, while specific, separate abilities might cause differing performance on divergent tests of cognitive processes. The CHC model 
addresses the influence of a general cognitive ability and separate cognitive abilities (McGrew, 2009), integrating intelligence and EF processes (Jewsbury, 2015).

The amount of variance explained in the intelligence indices in the young-adult sample was much lower than that explained in the models estimated in the child sample, and the variance explained in the inhibition measure and the set shifting measure was also lower in the young-adult sample than in the child sample, which is further evidence suggesting that those abilities differentiate and are determined by specific cognitive abilities in older populations. While the amount of variance explained in the observed EF measures was highly similar in both models, intelligence indices had more variance explained in the two-factor model than the onefactor model. Taken together, these results propose that differentiation of cognitive abilities happens across the EF and intelligence indices as lower amounts of variance in the observed variables was explained in the young-adult sample compared to the child sample in both models. The results also suggest that differentiation between EF and intelligence indices occurred with more variance explained in the intelligence indices in the two-factor model than in the one factor model in the young-adult sample. These findings highlight the differential nature of cognitive abilities (Brydges et al., 2012; Garrett, 1946), whereby these abilities become more distinguishable and stable with age (Brydges et al., 2014), especially in individuals with average and higher "general ability" levels, which allows for the variation and specialization among those general abilities of verbal and nonverbal intelligence indicative of Gc and Gf (Tucker-Drob, 2009). In other words, individuals with higher general ability have more room for the specialized cognitive abilities to differentiate and vary compared to individuals with lower levels of general cognitive ability whose cognitive resources are restricted. 
The only measure that showed an increase in variance explained in the young-adult sample compared to the child sample in the one-factor model was the indicator of updating (see Table 7). This finding is partially explained by the relation between updating and working memory, where updating has been strongly and consistently related to intelligence, with research questioning the validity of the updating construct as independent of what is measured by intelligence indices (see Jewsbury et al., 2015). As such, the latent general cognitive ability identified to underlie performance on intelligence and EF indices might have been more influential on updating tasks than other cognitive tasks.

The two models were also estimated with age-corrected WASI subtest scores in the young-adult sample, but, as expected, correcting for age in the WASI scores did not influence the fit of the models due to the absence of age-related effects on performance on all cognitive indices (see Table 4). These findings, along with the reduced amount of variance explained in cognitive indices in Models A and B in the young-adult sample speak to the differentiation and stability of cognitive abilities in this age group.

\section{Conclusions regarding differentiation and measurement of cognitive abilities. This}

attempt to examine the changing structure and organization of the underlying processes driving performance on measures of cognitive abilities (intelligence and EF) provided a better understanding of the role of latent abilities and associations among measurable processes. Previous research examining the relations between intelligence and EF report mixed results regarding the strength of the relations, depending on the types of measures used, and just as important, the approach used to score these measures. In both Ardila et al. (2000) and Rommelse et al. (2008), age-corrected intelligence test scores were used to examine their relations with EF. This practice is misleading because, as has been previously discussed and illustrated with the 
results here, there are documented age-related improvements in EF (e.g., Anderson 2002; Lehto et al., 2003; Salthouse 2005) and removing those effects from intelligence test scores will substantially reduce their associations with EF. Thus, it is crucial to understand the consequences of the procedures undertaken to measure these cognitive abilities and the way in which they are used in statistical analysis. Informed research should take into account the developmental nature of these processes and consider the implications of controlling age effects on the assessment of cognitive abilities.

Even with the very high inter-factor correlations presented in the two-factor models estimated and the good fit statistics of the one-factor models in both samples, the highest amount of variance accounted for in one of the cognitive indicators by the latent factors identified reached 59.1\% (see Table 7), which leaves a considerable proportion of variance unaccounted for. This finding indicates that these different measures of intelligence and EF have unique aspects that are not accounted for by a general cognitive ability factor or two separate factors of intelligence and EF in both samples. Therefore, the patterns in the results reflect the highly related nature of the separate cognitive variables rather than a complete overlap among those variables. This pattern is also proposed in Miyake and Friedman's (2012) model of a general executive ability underlying all EFs alongside multiple independent abilities belonging to each specific EF, and is similar to Anderson's (1992) theory of minimal cognitive architecture in the intelligence literature. The latter theory proposes that separate cognitive processes influence different domains of intellectual performance, but that these processes are limited by a single general ability which leads to associations across performance in all the different domains (i.e. different cognitive indices). This pattern is further supported by the $\mathrm{CHC}$ model of cognitive abilities (Carroll, 1993), which explains the role of broad abilities in explaining performance on 
narrow abilities. Yet, even though there are separate underlying processes between and within intelligence and EF indices, these large correlations have methodological and clinical implications which need to be considered, especially when examining related constructs.

\section{Cognitive Processes Predictive of Ratings of Inattention and Hyperactivity/Impulsivity}

The final analysis in this study tested whether the cognitive latent abilities predict ratings of inattention and hyperactivity/impulsivity. Furthermore, the analysis addressed implications of controlling for the relation between intelligence and EF which is sometimes done in the research literature but is also highly criticized, especially when the population studied differs in overall cognitive ability (see Dennis et al., 2009). It is reported here that the general cognitive ability factor significantly predicted parent ratings of inattention and hyperactivity/impulsivity in the child sample, while neither one of the two factors of intelligence and EF in the two-factor model uniquely predicted parent ratings of inattention and hyperactivity/impulsivity. In light of this finding, it is concluded that some of the shared processes underlying performance on intelligence tests and EF measures contributes to the development of controlling attention, behaviour, and impulses in children and adolescents. Yet, the amount of variance explained in parent ratings of inattention and hyperactivity/impulsivity was relatively small, 11.6\%. This result suggests either that other cognitive indices not utilized in this study, or other processes which are not determined by intelligence and $\mathrm{EF}$, also contribute to the development of attention and impulse control in childhood and adolescence. Nonetheless, the results support claims by Arffa (2007) and Dennis et al. (2009) regarding the effects of using intelligence test scores as a covariate in clinical and developmental studies where one would risk removing the reliable variance in EF that would predict outcomes or differentiate populations. 
In the young-adult sample, none of the factors significantly predicted ratings of inattention and hyperactivity/impulsivity across the two models. This finding was not surprising considering that ratings of inattention and hyperactivity/impulsivity did not initially correlate with any of the cognitive indices. It is difficult to reach conclusions regarding the role of intelligence and $\mathrm{EF}$ in attention and impulse control in this sample, but cognitive abilities and attention and hyperactivity/impulsivity in the young-adult population are expected to be relatively more differentiated and well-developed, especially because performance on these measures was less dependent on age than it was in the child sample.

\section{Summary of Conclusions and Implications}

The results provide insight on the changing relations and underlying cognitive processes among some of the most utilized measures of cognitive ability (Miyake \& Friedman, 2012). Consistent with previous research and theory, cognitive indices of intelligence and EF showed age-related improvements in the child sample and higher stability in the young-adult sample (Cattell, 1963; Deary et al., 2000; Salthouse \& Davis, 2006; Jurado \& Rosselli, 2007). Not only are age effects different across different periods, but also the pattern of relations among different cognitive indices varied across samples.

Across all analyses conducted, results consistently found higher relations among scores on intelligence and EF indices in the child sample compared to the young-adult sample and are consistent with cross-sectional results from $\mathrm{Li}$ et al. (2004). These findings reflect the developing nature of childhood and adolescence periods and the larger role of an underlying general cognitive ability in those periods than in later, more stable developmental periods, such as young adulthood, where separate cognitive abilities (rather than a domain-free general ability) are better 
determinants of performance across separate cognitive tasks. This pattern raises concerns regarding our reliance on composite intelligence scores which are used to reflect general intellectual functioning in young adults. Even though the one-factor and two-factor models were not significantly different in the young-adult sample, considering the amount of variance explained in the cognitive indices and taking into consideration results from the literature (e.g., Tucker-Dub, 2009), the results of this study suggest that while composite scores might appropriately represent children's developing cognitive abilities, using composite scores with young adults might be inadequate, especially with higher scoring young adults and middle-aged adults because their separate cognitive abilities would be more distinct than related.

With typically developing young adults and middle-aged adults, there is more room for specialization of separate cognitive abilities because they are not represented by an overly simplistic general, domain-free cognitive mechanism, where cognitive abilities have fully developed and are not a function of age. On the other hand, populations with lower overall cognitive functioning or ability, such as populations with neurodevelopmental disorders (Dennis et al., 2009), present stronger relations among the different cognitive abilities hypothesized to underlie performance on cognitive tasks than in typically developing populations. This pattern is supported by findings which present stronger correlations among cognitive indices in low intellectual-ability groups compared to average and high intellectual-ability groups (Deary et al., 1996; Kane, Oakland, \& Brand, 2006; Reynolds \& Keith, 2007). Similarly, with children and adolescents, performance across different cognitive tasks is highly related and dependent on age, thus rendering commonly practiced statistical control of intelligence when measuring or assessing EF misguided, as illustrated in our analysis. The two factors of intelligence and EF in the child sample were non-significant unique predictors of parent ratings of inattention and 
hyperactivity/impulsivity. Yet, the general cognitive ability factor was a significant predictor of parent ratings of inattention and hyperactivity/impulsivity. It is concluded that the general cognitive ability underlying performance on measures of intelligence and EF has an important role in the development of attention control and behavioural control and thus it is inappropriate to control or partial out the variance shared between intelligence and EF when examining their effects on outcomes, especially in populations where these cognitive abilities are still developing, and as such are highly related, supporting arguments by Dennis et al. (2009) and Arffa (2007). Thus, measurement and analysis have implications for identifying potential targets for intervention, such as EF, in clinical populations.

\section{Future Directions and Limitations}

One of the noteworthy findings in this study is the changing pattern of relations among the cognitive indices across samples. Because these changing relations across developmental periods have been documented in previous work (Li et al., 2004; Salthouse \& Davis, 2006; Tucker-Drob, 2009), and presented in this work as well, it is important to examine the changing relations in clinical populations of different ages. Doing so would have important implications for our understanding of cognitive trajectories across diverse populations (e.g. Rajendran et al., 2013), especially with the current theoretical understanding of the limitations of low overall cognitive ability. The next step is to support these results with longitudinal data to examine the changing pattern of relations among cognitive indices of both intelligence and EF in the same sample of participants. Measuring the changing relations among these processes in the same participants over time would provide more insight and concrete conclusions than those provided by cross-sectional data alone. 
It is also important to replicate the results regarding the underlying cognitive abilities with a larger set of measures. In this study, five commonly utilized and representative measures of cognitive abilities were used, but there are many more cognitive ability measures utilized in clinical and research settings which might reflect different dimensions and processes than the ones used in the current models. Another promising direction is to test the same models with a variety of different outcomes, scoping numerous domains. As discussed previously, intelligence and EF predict an array of outcomes, and it would be important to identify the outcomes that are predicted uniquely by separate cognitive processes and the ones predicted by shared cognitive, or general, processes and the implications thereafter on assessment and intervention.

This study provides unique findings with important implications, but it is not without its limitations. The briefly discussed CHC model of cognitive abilities (Carroll, 1993) actually includes 16 interrelated cognitive abilities which are encompassed by a general cognitive ability factor. Yet, this study focused mainly on indicators of nonverbal and verbal intelligence (Gf and Gc, respectively) and three of the EF processes, limiting the conclusions regarding broader aspects of cognitive abilities which include processing speed, visual processing, auditory processing, quantitative knowledge, and many more. However, the intelligence indicators used have been correlated with the Wechsler Intelligence Scale for Children $(r=.81)$ and the Wechsler Adult Intelligence Scale $(r=.87)$, and are considered appropriate indicators of general intelligence (Steinberg et al., 2009), and the three EFs tested are among the most examined and based upon the most replicated EF structure (Miyake et al., 2000). As mentioned, five measures of cognitive abilities were used as representative of intelligence and EF constructs despite the presence of more potential cognitive measures, which might result in different factor structures. Therefore, it is important to evaluate the conclusions stated within the broader literature and 
previous documented findings. It would be best for future projects to use two or more indicators to represent each cognitive construct in the model. This study also utilized one measure of inattention and hyperactivity/impulsivity per sample. Future studies should have a multiinformant assessment of cognitive and behavioural regulation for a more reliable and valid representation of these constructs. A final limitation concerns the updating measures used that were different across samples. This difference is a concern for the direct comparison of relations between updating and other cognitive abilities across samples, and should be addressed in further replications of this work. Yet, the results concerning the relations between updating and other measures were consistent across samples and among the separate analyses in each sample and are supported by previous work finding high associations between updating measures and intelligence indices (e.g. Belacchi et al., 2010; Engle, 1999; Salthouse \& Pink, 2008).

In conclusion, the results of the analyses suggest that many cognitive abilities (including intelligence and EFs) show higher dependence on age in younger populations than older populations, where it seems that it is as important to consider the high relations among cognitive abilities in typically developing child samples as it is in atypically developing child samples. The results obtained from the analyses conducted in this study supported the developmental nature of cognitive abilities in younger populations, and highlighted the differences in the patterns of relations of cognitive abilities between a child and a young-adult sample along with the implications thereafter on cognitive and neuropsychological assessment in children and adolescents compared to young adults. 


\section{References}

Ackerman, P. L., \& Kanfer, R. (2004). Cognitive, affective, and conative aspects of adult intellect within a typical and maximal performance framework.Motivation, emotion, and cognition: Integrative perspectives on intellectual functioning and development, 119-141.

Anderson, M. (1992). Intelligence and development: A cognitive theory. Blackwell Publishing.

Anderson, M. (2001). Annotation: Conceptions of intelligence. Journal of Child Psychology and Psychiatry, 42(3), 287-298.

Anderson, P. (2002). Assessment and development of executive function (EF) during childhood. Child Neuropsychology, 8, 71-82.

Ardila, A., Pineda, D., \& Rosselli, M. (2000). Correlation between intelligence test scores and executive function measures. Archives of clinical neuropsychology, 15(1), 31-36.

Arffa, S. (2007). The relationship of intelligence to executive function and non-executive function measures in a sample of average, above average, and gifted youth. Archives of Clinical Neuropsychology, 22(8), 969-978.

Baddeley, A. (2000). The episodic buffer: a new component of working memory?. Trends in cognitive sciences, 4(11), 417-423.

Baddeley, A. D., \& Hitch, G. J. (1994). Developments in the concept of working memory. Neuropsychology, 8(4), 485.

Baumeister, R. F. (2002). Ego depletion and self-control failure: An energy model of the self's executive function. Self and Identity, 1(2), 129-136. 
Baumeister, R. F., Heatherton, T. F., \& Tice, D. M. (1994). Losing control: How and why people fail at self-regulation. Academic Press

Baumeister, R. F., Vohs, K. D., \& Tice, D. M. (2007). The strength model of selfcontrol. Current directions in psychological science, 16(6), 351-355.

Belacchi, C., Carretti, B., \& Cornoldi, C., (2010). The role of working memory and updating in Coloured Raven Matrices performance in typically developing children. European Journal of Cognitive Psychology, 22:7,1010-1020.

Benedek, M., Jauk, E., Sommer, M., Arendasy, M., \& Neubauer, A. C. (2014). Intelligence, creativity, and cognitive control: the common and differential involvement of executive functions in intelligence and creativity. Intelligence, 46, 73-83.

Best, J. R., Miller, P. H. \& Jones, L. L. (2009). Executive functions after age 5: changes and correlates. Developmental Review, 29, 180-200.

Best, J. R., Miller, P. H. \& Naglieri, J. A. (2011). Relations between executive function and academic achievement from ages 5 to 17 in a large, representative national sample. Learning and Individual Differences, 21, 327-336.

Biederman, J., Monuteaux, M. C., Doyle, A. E., Seidman, L. J., Wilens, T. E., Ferrero, F., ... \& Faraone, S. V. (2004). Impact of executive function deficits and attentiondeficit/hyperactivity disorder (ADHD) on academic outcomes in children. Journal of consulting and clinical psychology, 72(5), 757.

Borella, E., Carretti, B., \& Pelegrina, S. (2010). The specific role of inhibition in reading comprehension in good and poor comprehenders. Journal of Learning Disabilities, 43(6), 
541-552.

Brydges, C. R., Fox, A. M., Reid, C. L., \& Anderson, M. (2014). The differentiation of executive functions in middle and late childhood: A longitudinal latent-variable analysis. Intelligence, 47, 34-43.

Brydges, C. R., Reid, C. L., Fox, A. M., \& Anderson, M. (2012). A unitary executive function predicts intelligence in children. Intelligence, 40(5), 458-469.

Carlson, Zelazo, \& Faja (2012). Executive Functions. Chapter 25 in Zelazo, P. D. (2013). The Oxford Handbook of Developmental Psychology, Vol. 1: Body and Mind (Vol. 1). Oxford University Press.

Carroll, J.B. (1993). Human cognitive abilities: A survey of factor-analytic studies. New York: Cambridge University Press.

Carroll, J. B. (1997). The three-stratum theory of cognitive abilities. In D. P. Flanagan, \& P. L. Harrison (Eds.), Contemporary intellectual assessment: Theories, tests and issues. (pp. 122-130). New York: Guilford.

Casey, B. J., Tottenham, N., \& Fossella, J. (2002). Clinical, imaging, lesion, and genetic approaches toward a model of cognitive control. Developmental psychobiology, 40(3), 237-254.

Cattell, R. B. (1963). Theory of fluid and crystallized intelligence: A critical experiment. Journal of Educational psychology, 54(1), 1.

Cattell, R. B. (1971). Abilities: Their structure, growth and action. Boston, MA: HoughtonMifflin. 
Clark, C., Prior, M. \& Kinsella, G. (2002). The relationship between executive function abilities, adaptive behaviour, and academic achievement in children with externalising behaviour problems. Journal of Child Psychology and Psychiatry, 43, 785-796.

Conway, A. R., Kane, M. J., \& Engle, R. W. (2003). Working memory capacity and its relation to general intelligence. Trends in cognitive sciences, 7(12), 547-552.

Cunningham, W. A., \& Zelazo, P. D. (2007). Attitudes and evaluations: a social cognitive neuroscience perspective. Trends in Cognitive Sciences, 11 , 97-104.

Dang, C. P., Braeken, J., Ferrer, E., \& Liu, C. (2012). Unitary or non-unitary nature of working memory? Evidence from its relation to general fluid and crystallized intelligence. Intelligence, 40(5), 499-508.

Deary, I. J., Egan, V., Gibson, G. J., Austin, E. J., Brand, C. R., \& Kellaghan, T. (1996). Intelligence and the differentiation hypothesis. Intelligence, 23(2), 105-132.

Deary, I. J., Penke, L., \& Johnson, W. (2010). The neuroscience of human intelligence differences. Nature Reviews Neuroscience, 11(3), 201-211.

Deary, I. J., Strand, S., Smith, P., \& Fernandes, C. (2007). Intelligence and educational achievement. Intelligence, 35(1), 13-21.

Deary, I. J., Whalley, L. J., Lemmon, H., Crawford, J. R., \& Starr, J. M. (2000). The stability of individual differences in mental ability from childhood to old age: follow-up of the 1932 Scottish Mental Survey. Intelligence, 28(1), 49-55. 
Dennis, M., Francis, D. J., Cirino, P. T., Schachar, R., Barnes, M. A., \& Fletcher, J. M. (2009). Why IQ is not a covariate in cognitive studies of neurodevelopmental disorders. Journal of the International Neuropsychological Society, 15(03), 331-343.

Diamond, A. (2013). Executive functions. The Annual Review of Psychology, 64, 135-168.

Diamond, A., \& Lee, K. (2011). Interventions shown to aid executive function development in children 4 to 12 years old. Science, 333(6045), 959-964.

Duckworth, A. L., Quinn, P. D., \& Tsukayama, E. (2012). What No Child Left Behind leaves behind: The roles of IQ and self-control in predicting standardized achievement test scores and report card grades. Journal of Educational Psychology, 104(2), 439.

Duckworth, A. L., Tsukayama, E., \& May, H. (2010). Establishing causality using longitudinal hierarchical linear modeling: An illustration predicting achievement from selfcontrol. Social psychological and personality science.

Duncan, J., Seitz, R. J., Kolodny, J., Bor, D., Herzog, H., Ahmed, A., ... \& Emslie, H. (2000). A neural basis for general intelligence. Science, 289(5478), 457-460.

Engle, R. W., Tuholski, S. W., Laughlin, J. E., \& Conway, A. R. (1999). Working memory, short-term memory, and general fluid intelligence: a latent-variable approach. Journal of experimental psychology: General, 128(3), 309.

Friedman, N. P., Haberstick, B. C., Willcutt, E. G., Miyake, A., Young, S. E., Corley, R. P., \& Hewitt, J. K. (2007). Greater attention problems during childhood predict poorer executive functioning in late adolescence. Psychological Science, 18, 893-900. 
Friedman, N. P., Miyake, A., Corley, R. P., Young, S. E., DeFries, J. C., \& Hewitt, J. K. (2006). Not all executive functions are related to intelligence. Psychological science, 17(2), 172179.

Friedman, N. P., Miyake, A., Young, S. E., DeFries, J. C., Corley, R. P., \& Hewitt, J. K. (2008). Individual differences in executive functions are almost entirely genetic in origin. Journal of Experimental Psychology: General, 137(2), 201.

Giofrè, D., \& Mammarella, I. C. (2014). The relationship between working memory and intelligence in children: Is the scoring procedure important? Intelligence, 46, 300-310.

Gioia, G. A., Isquith, P. K., Guy, S. C., \& Kenworthy, L. (2000). Test review behavior rating inventory of executive function. Child Neuropsychology, 6(3), 235-238.

Gogtay, N., Giedd, J. N., Lusk, L., Hayashi, K. M., Greenstein, D., Vaituzis, A. C., ... \& Thompson, P. M. (2004). Dynamic mapping of human cortical development during childhood through early adulthood. Proceedings of the National Academy of Sciences of the United States of America, 101(21), 8174-8179.

Gottardo, A., Stanovich, K. E., \& Siegel, L. S. (1996). The relationships between phonological sensitivity, syntactic processing, and verbal working memory in the reading performance of third-grade children. Journal of Experimental Child Psychology, 63, 563-582.

Gottfredson, L. S. (1997). Mainstream science on intelligence: An editorial with 52 signatories, history, and bibliography. Intelligence, 24(1), 13-23.

Gerenberg, M. T. (2006). Promoting resilience in children and youth: Preventive interventions and their interface with neurescience. New York Academy Of Seciences, 1094, 139-150. 
Hofmann, W., Schmeichel, B. J., \& Baddeley, A. D. (2012). Executive functions and selfregulation. Trends in cognitive sciences, 16(3), 174-180.

Horn, J. L., \& Noll, J. (1997). Human cognitive capabilities: Gf-Gc theory. In D. P. Flanagan, J. L. Genshaft, \& P. L. Harrison (Eds.), Contemporary intellectual assessment: Theories, tests and issues (pp. 53-91). New York: Guilford.

Hu, L. T., \& Bentler, P. M. (1999). Cutoff criteria for fit indexes in covariance structure analysis: Conventional criteria versus new alternatives. Structural equation modeling: a multidisciplinary journal, 6(1), 1-55.

Huizinga, M., Dolan, C. V., \& van der Molen, M. W. (2006). Age-related change in executive function: Developmental trends and a latent variable analysis.Neuropsychologia, 44(11), 2017-2036.

Jewsbury, P. A., Bowden, S. C., \& Strauss, M. E. (2015). Integrating the Switching, Inhibition, and Updating Model of Executive Function With the Cattell—Horn—Carroll Model.

Jurado, M. B., \& Rosselli, M. (2007). The elusive nature of executive functions: a review of our current understanding. Neuropsychology review, 17(3), 213-233.

Kane, H. D., Oakland, T. D., \& Brand, C. R. (2006). Differentiation at higher levels of cognitive ability: Evidence from the United States. The Journal of genetic psychology, 167(3), 327341.

Kane, M. J., \& Engle, R. W. (2002). The role of prefrontal cortex in working-memory capacity, executive attention, and general fluid intelligence: An individual-differences perspective. Psychonomic bulletin \& review, 9(4), 637-671. 
Kane, M. J., Hambrick, D. Z., \& Conway, A. R. (2005). Working memory capacity and fluid intelligence are strongly related constructs: comment on Ackerman, Beier, and Boyle (2005).

Kline, R. B. (2011). Convergence of structural equation modeling and multilevel modeling. na.

Lehto, J. E., Juujärvi, P., Kooistra, L., \& Pulkkinen, L. (2003). Dimensions of executive functioning: Evidence from children. British Journal of Developmental Psychology, 21(1), $59-80$

Lezak, M. (2004). Neuropsychological assessment (4th ed.). New York, NY: Oxford University Press.

Li, S. C., Lindenberger, U., Hommel, B., Aschersleben, G., Prinz, W., \& Baltes, P. B. (2004). Lifespan transformations in the couplings of mental abilities and underlying cognitive processes. Psychological Science, 15(3), 155-163.

Mayes, S. D., Calhoun, S. L., Bixler, E. O., \& Zimmerman, D. N. (2009). IQ and neuropsychological predictors of academic achievement. Learning and Individual Differences, 19(2), 238-241.

McGrew, K. S. (2009). CHC theory and the human cognitive abilities project: Standing on the shoulders of the giants of psychometric intelligence research. Intelligence, 37(1), 1-10.

McGrew, K. S. (2005). The Cattell-Horn-Carroll theory of cognitive abilities. In D. P. Flanagan, \& P. L. Harrison (Eds.),Contemporary intellectual assessment: Theories, tests, and issues (pp. 136-181)., 2nd ed. New York: Guilford Press. 
McGrew, K. S. (1997). Analysis of the major intelligence batteries according to a proposed comprehensive Gf-Gc framework. In D. P. Flanagan, J. L. Genshaft, \& P. L. Harrison (Eds.), Contemporary intellectual assessment: Theories, tests, and issues (pp. 151-179). New York: Guilford.

Miller, E. K., \& Cohen, J. D. (2001). An integrative theory of prefrontal cortex function. Annual review of neuroscience, 24(1), 167-202.

Miller, M. R., Giesbrecht, G. F., Müller, U., McInerney, R. J., \& Kerns, K. A. (2012). A latent variable approach to determining the structure of executive function in preschool children. Journal of Cognition and Development, 13(3), 395-423.

Miyake, A., \& Friedman, N. P. (2012). The nature and organization of individual differences in executive functions four general conclusions. Current directions in psychological science, 21(1), 8-14.

Miyake, A., Friedman, N. P., Emerson, M. J., Witzki, A. H., Howerter, A., \& Wager, T. D. (2000). The unity and diversity of executive functions and their contributions to complex "frontal lobe" tasks: A latent variable analysis.Cognitive psychology, 41(1), 49-100.

Moffitt, T. E., Arseneault, L., Belsky, D., Dickson, N., Hancox, R. J., Harrington, H., ... \& Caspi, A. (2011). A gradient of childhood self-control predicts health, wealth, and public safety. Proceedings of the National Academy of Sciences, 108(7), 2693-2698.

Nisbett, R. E., Aronson, J., Blair, C., Dickens, W., Flynn, J., Halpern, D. F., \& Turkheimer, E. (2012). Intelligence: new findings and theoretical developments.American psychologist, 67(2), 130. 
Pennington, B. F., \& Ozonoff, S. (1996). Executive functions and developmental psychopathology. Journal of child psychology and psychiatry, 37(1), 51-87.

Protopapas, A., Archonti, A., \& Skaloumbakas, C. (2007). Reading ability is negatively related to Stroop interference. Cognitive Psychology, 54(3), 251-282.

Rajendran, K., Rindskopf, D., O’Neill, S., Marks, D. J., Nomura, Y., \& Halperin, J. M. (2013). Neuropsychological functioning and severity of ADHD in early childhood: A four-year cross-lagged study. Journal of abnormal psychology, 122(4), 1179.

Regard, M. (1981). Cognitive rigidity and flexibility: A neuropsychological study. Unpublished Ph.D. dissertation, University of Victoria.

Reitan, R. M. (1955). The relation of the Trail Making Test to organic brain damage. Journal of Consulting Psychology, 19, 393-394.

Reitan, R. M. (1958). Validity of the Trail Making Test as an indicator of organic brain damage. Perceptual and Motor Skills, 8, 271-276.

Reynolds, M. R., \& Keith, T. Z. (2007). Spearman's law of diminishing returns in hierarchical models of intelligence for children and adolescents.Intelligence, 35(3), 267-281.

Rommelse, N. N. J., Altink, M. E., Oosterlaan, J., Buschgens, C. J. M., Buitelaar, J., \& Sergeant, J. A. (2008). Support for an independent familial segregation of executive and intelligence endophenotypes in ADHD families.Psychological medicine, 38(11), 1595.

Rosseel, Y. (2012). lavaan: An R package for structural equation modeling. Journal of Statistical Software, 48(2), 1-36. 
Salthouse, T. A. (2005). Relations between cognitive abilities and measures of executive functioning. Neuropsychology, 19(4), 532.

Salthouse, T. A., Atkinson, T. M., \& Berish, D. E. (2003). Executive functioning as a potential mediator of age-related cognitive decline in normal adults. Journal of Experimental Psychology: General, 132(4), 566.

Salthouse, T. A., \& Davis, H. P. (2006). Organization of cognitive abilities and neuropsychological variables across the lifespan. Developmental Review,26(1), 31-54.

Salthouse, T. A., \& Pink, J. E. (2008). Why is working memory related to fluid intelligence? Psychonomic Bulletin \& Review, 15(2), 364-371.

Sattler, J. M. (2008). Assessment of children: Cognitive foundations. JM Sattler.

Sbicigo, B. J., Piccolo, D. L., Fonseca, P. R., \& de Salles, F. J. (2014). Working memory and fluid intelligence: the role executive processes, age and school type in children. Universitas Psychologica, 13(3).

Siegler, R. S., Duncan, G. J., Davis-Kean, P. E., Duckworth, K., Claessens, A., Engel, M., ... \& Chen, M. (2012). Early predictors of high school mathematics achievement. Psychological science, 23(7), 691-697.

Skirbekk, V. (2004). Age and individual productivity: A literature survey. Vienna yearbook of population research, 133-153.

Spearman C. "General intelligence” objectively determined and measured. American Journal of Psychology 1904;15:201-293. 
Sternberg, R. J., \& Kaufman, J. C. (1998). Human abilities. Annual Review of Psychology, 49, $1134-1139$.

Strayhorn, J. M. (2002). Self-control: Theory and research. Journal of the American Academy of Child \& Adolescent Psychiatry, 41(1), 7-16.

Swanson, J. M., Schuck, S., Porter, M., Carlson, C., Hartman, C., Sergeant, J. A., ... \& Wigal, T. (2012). Categorical and Dimensional Definitions and Evaluations of Symptoms of ADHD: History of the SNAP and the SWAN Rating Scales. International Journal of Educational and Psychological Assessment, 10, 51-70.

Tangney, J. P., Baumeister, R. F., \& Boone, A. L. (2004). High self-control predicts good adjustment, less pathology, better grades, and interpersonal success. Journal of personality, 72(2), 271-324.

Tucker-Drob, E. M. (2009). Differentiation of cognitive abilities across the life span. Developmental psychology, 45(4), 1097.

van der Sluis, S., de Jong, P. F., \& van der Leij, A. (2007). Executive functioning in children, and its relations with reasoning, reading, and arithmetic.Intelligence, 35(5), 427-449.

Watkins, M. W., Lei, P. W., \& Canivez, G. L. (2007). Psychometric intelligence and achievement: A cross-lagged panel analysis. Intelligence, 35(1), 59-68.

Weaver, E. A., \& Stewart, T. R. (2012). Dimensions of judgment: Factor analysis of individual differences. Journal of Behavioral Decision Making, 25(4), 402-413. 
Wechsler (1999). Wechsler Abbreviated Scale of Intelligence (WASI). The Psychological Corporation.

Wiebe, S. A., Sheffi eld, T., Nelson, J. M., Clark, C. A. C., Chevalier, N., \& Espy, K.. (2011). The structure of executive function in 3-year-olds. Journal of Experimental Child Psychology, 108, 436-452.

Xu, F., Han, Y., Sabbagh, M. A., Wang, T., Ren, X., \& Li, C. (2013). Developmental differences in the structure of executive function in middle childhood and adolescence. PloS one, $8(10)$, e77770.

Yuan, K. H., \& Bentler, P. M. (2000). Three likelihood-based methods for mean and covariance structure analysis with nonnormal missing data. Sociological methodology, 30, 165-200.

Zelazo, P. D. (2004). The development of conscious control in childhood.Trends in cognitive sciences, $8(1), 12-17$.

Zelazo, P. D., Craik, F. I., \& Booth, L. (2004). Executive function across the life span. Acta Psychologica, 115(2), 167-183.

Zelazo, P. D., \& Mu"ller, U. (2010). Executive function in typical and atypical development. In U. Goswami (Ed.), Blackwell handbook of childhood cognitive development (2nd ed., pp. 574-603). Malden, MA: Blackwell 